Doi 10.5943/sif/3/1/17

Copyright ( $)$ Mushroom Research Foundation

\title{
Taxonomic circumscription and phylogenetics of novel didymellaceous taxa with brown muriform spores
}

\author{
Wanasinghe $\mathrm{DN}^{1,2,3}$, Jeewon $\mathrm{R}^{4}$, Peršoh $\mathrm{D}^{5}$, Jones $\mathrm{EBG}^{6}$, Camporesi $\mathrm{E}^{7,8,9}$, \\ Bulgakov TS ${ }^{10}$, Gafforov $\mathrm{YS}^{11}$ and Hyde $\mathrm{KD}^{1,2,3 *}$
}

${ }^{1}$ Key Laboratory for Plant Biodiversity and Biogeography of East Asia (KLPB), Kunming Institute of Botany, Chinese Academy of Science, Kunming 650201, Yunnan China

${ }^{2}$ Center of Excellence in Fungal Research, Mae Fah Luang University, Chiang Rai, 57100, Thailand

${ }^{3}$ World Agro Forestry Centre, East and Central Asia, 132 Lanhei Road, Kunming 650201, Yunnan China

${ }^{4}$ Department of Health Sciences, Faculty of Science, University of Mauritius, Reduit, Mauritius

${ }^{5}$ AG Geobotany, Faculty of Biology and Biotechnology, Ruhr-Universitat Bochum, Universitatsstraße 150, 44801

Bochum, Germany;

${ }^{6}$ Nantgaredig, 33B St. Edwards Road, Southsea, Hants., PO5 3DH, UK;

${ }^{7}$ Società per gli Studi Naturalistici della Romagna, C.P. 144, Bagnacavallo (RA), Italy;

${ }^{8}$ A.M.B. Gruppo Micologico Forlivese "Antonio Cicognani”, Via Roma 18, Forlì, Italy;

${ }^{9}$ A.M.B. Circolo Micologico "Giovanni Carini”, C.P. 314, Brescia, Italy;

${ }^{10}$ Russian Research Institute of Floriculture and Subtropical Crops, Sochi, 354002, Yana Fabritsiusa street, 2/28, Krasnodar region, Russia;

${ }^{11}$ Laboratory of Mycology, Institute of Botany, Academy of Sciences of the Republic of Uzbekistan, 32 Durmon yuli Street, Tashkent 100125, Uzbekistan

Wanasinghe DN, Jeewon R, Peršoh D, Jones EBG, Camporesi E, Bulgakov TS, Gafforov YS, Hyde KD 2018 - Taxonomic circumscription and phylogenetics of novel didymellaceous taxa with brown muriform spores. Studies in Fungi 3(1), 152-175, Doi 10.5943/sif/3/1/17

\section{Abstract}

Sexual morph of didymellaceous taxa are characterized by their ascomata with relatively thin peridium, cylindric-clavate to clavate, short-pedicellate or apedicellate asci, hyaline to brown, 1septate to muriform ascospores. Its asexual morphs are coelomycetous and comprising pycnidial or acervulus conidiomata, phialidic, hyaline conidiogenous cells and hyaline or pale brown, septate or aseptate conidia. The majority of these cosmopolitan species are plant associated fungi which can be pathogens on a wide range of hosts and some species are of particular relevance for quarantine measures. Recent studies have significantly improved the taxonomy and systematics of didymellaceous taxa based on molecular phylogenetics. In contrast to the accurate and detailed studies on the asexual morphs which are common obligate pathogens, information on their usually saprobic sexual morphs is still limited. Among these phenotypically diverse species, spore characteristics are quite unique as most have hyaline spores with 0-1 septum, while only Neomicrosphaeropsis and Didymellocamarosporium are reported as producing pigmented, muriform spores. These dematiaceous muriform spores are characteristic of a considerable number of species that may be quite divergent in other characters. During taxonomic investigations on the diversity of didymellaceous taxa, we have isolated species from Alhagi pseudalhagi, Coronilla emerus, Cytisus sp., Elaeagnus angustifolia and Spartium junceum in Italy, Russia and Uzbekistan. A comprehensive phylogeny, based on four loci (ITS, LSU, rpb2 and tub2) is used to infer species relationships. Comprehensive morphological descriptions and in-depth phylogenetic investigations of five new species viz. Ascochyta coronillae-emeri, Microsphaeropsis spartii-juncei, Neomicrosphaeropsis alhagi-pseudalhagi, N. cytisicola and N. elaeagni are presented. 
Keywords - five new species - coelomycetes - Italy - multi-gene - phylogeny - Pleosporales Russia - saprobic - taxonomic-ambiguity - Uzbekistan

\section{Introduction}

The family Didymellaceae was proposed by de Gruyter et al. (2009) to accommodate phomalike taxa, viz. Ascochyta, Didymella and Phoma, which probably diverged in the Jurassic or earlier from an ancestor whose origin can be estimated about 63 mya (crown age) or 115 mya (stem age) ago (Liu et al. 2017). Didymellaceae is one of the most species-rich families in the fungal kingdom and includes 4956 and 4713 taxon epithets listed in MycoBank and Index Fungorum, respectively (2017). More than 50\% from the total epithets are listed as Phoma and over 30\% are recorded as Ascochyta. In a recent study, Chen et al. (2017) revised Didymellaceae and improved our understanding of their distribution and biodiversity. They have proposed 19 genera in the family and currently the family comprises 31 genera, including Cumuliphoma, Didymellocamarosporium, Didysimulans, Ectophoma, Endocoryneum, Juxtiphoma, Neodidymella, Pseudoascochyta, Pseudohendersonia, Remotididymella, Similiphoma and Vacuiphoma (Ariyawansa et al. 2015, Crous et al. 2016, Wijayawardene et al. 2016, 2018, Tibpromma et al. 2017, Valenzuela-Lopez et al. 2018). The majority of members in Didymellaceae are plant associated fungi which can be pathogens on a wide range of hosts, largely causing leaf and stem lesions, with some of particular relevance for quarantine measures (Aveskamp et al. 2008, 2010, Chen et al. 2015, 2017). Didymellaceae are cosmopolitan and able to adapt to extreme environmental conditions i.e. temperature, nutrients, moisture, absolute darkness and they can grow in exposed habitats such as air, soil, water, limestone from caves (Chen et al. 2017) and inorganic materials including asbestos, cement and paint (Aveskamp et al. 2008). Given their ubiquitous nature, additional taxonomic and ecological knowledge are prerequisites to understand their biology and their significance in the environment, especially in agriculture.

In contrast to the accurate and detailed studies on their asexual morphs, information is still limited on their sexual morphs, which usually grow as saprobes, in contrast to their pathogenic asexual counterparts (Chen et al. 2017). Determining the phylogenetic placement of sexual morphs is crucial to properly define the taxonomic boundaries within the polyphyletic and morphologically homogeneous genera (i.e. Ascochyta, Didymella and Phoma). Knowledge of the sexual-asexual relationships will considerably improve our understanding of many of the specific biological features. Of the 28 genera in this family, sexual morphs are known for 12 genera (Jayasiri et al. 2017) and their ascospores are mostly hyaline and 1-septate. There is only one sexual morph recorded in this family with pigmented muriform spores, Neomicrosphaeropsis tamaricicola $(=$ Phoma tamaricicola), introduced by Crous et al. (2014). Pigmented muriform spores are characteristic for a considerable number of species being divergent in other characters. For asexual morphs, Didymellocamarosporium tamaricis (Wijayawardene et al. 2016) is the only asexual member recorded with pigmented muriform conidia in this family.

We are investigating the diversity of microfungi that produce brown, muriform spores with the aim of clarifying their taxonomy based on morphology coupled with multigene phylogeny (Wanasinghe et al. 2014a, b, 2015, 2016, 2017a, b, 2018). As part of this study, we have isolated taxa from Alhagi pseudalhagi, Coronilla emerus, Cytisus sp., Elaeagnus angustifolia and Spartium junceum species in Italy, Russia and Uzbekistan which belong to the family Didymellaceae. Here we present comprehensive morphological descriptions and in-depth phylogenetic investigation of those taxa.

\section{Materials and Methods}

\section{Sampling, examination and isolation}

The novel strains were isolated from Alhagi pseudalhagi, Coronilla emerus, Cytisus sp., Elaeagnus angustifolia and Spartium junceum in Italy and Russia. Uzbekistan specimens were loaned from Tashkent Mycological Herbarium (TASM) of the Institute of Botany, Academy of 
Sciences of Uzbekistan, Tashkent. These collections were examined and isolated following the methods used by Wanasinghe et al. (2017a). Type and isotype specimens of new species in this study are deposited in the Mae Fah Luang University (MFLU) Herbarium. Living cultures are deposited at the Culture Collection of Mae Fah Luang University (MFLUCC) and duplicated in International Collection of Microorganisms from Plants (ICMP), Landcare Research, Auckland, New Zealand.

\section{DNA isolation, amplification and phylogenetic analyses}

Total genomic DNA was extracted from fresh mycelia using the protocol described by Wanasinghe et al. (2017a). When fungi failed to grow in culture, DNA was extracted directly from ascomycete fruiting bodies by following the protocol described by Wanasinghe et al. (2018). DNA to be used as template for PCR were stored at $4{ }^{\circ} \mathrm{C}$ for use in regular work and duplicated at $-20{ }^{\circ} \mathrm{C}$ for long term storage. The primers ITS5 and ITS4 (White et al. 1990) were used to amplify part of rDNA 18S ( $3^{\prime}$ end), the first internal transcribed spacer (ITS1), the 5.8S rRNA gene, the second ITS region (ITS2), and part of the 28S rRNA (5' end); the primers LR0R (Rehner \& Samuels 1994), LR5 (Vilgalys \& Hester 1990) were used for LSU amplification; Btub2Fd and Btub4Rd (Woudenberg et al. 2009) for the partial $\beta$-tubulin (tub2) gene region, and RPB2-5F (Sung et al. 2007) and fRPB2-7cR (Liu et al. 1999) for the RNA polymerase II second largest subunit (rpb2). Amplicons for ITS and LSU locus were generated following the protocols listed in Wanasinghe et al. (2017a) and the protocols of Chen et al. (2015) were used to amplify tub2 and rpb2.

Sequencing was conducted in both directions with the same primer pair used for amplification at BGI, Ltd., Shenzhen, P.R. China. Consensus sequences were assembled in BioEdit v. 7.0.5.2 (Hall 1999) and additional reference sequences were obtained from GenBank (Table 1). Subsequent alignments for each locus were generated with MAFFT v. 7 (http://mafft.cbrc.jp/alignment/server/index.html; Kuraku et al. 2013, Katoh et al. 2017), and manually corrected when necessary in BioEdit v7.0.9 (Hall 1999). Each locus and the concatenated aligned dataset were analysed separately using Maximum Likelihood (ML), Maximum Parsimony (MP) and Bayesian Inference (BI). The best-fit models of evolution for the four loci tested (GTR+I+G for all gene regions) were estimated by MrModeltest v. 2.3 (Nylander 2004).

Parsimony analysis was carried out with the heuristic search option in PAUP (Phylogenetic Analysis Using Parsimony) v. $4.0 \mathrm{~b} 10$ with the following parameter settings: characters unordered with equal weight, random taxon addition, branch swapping with tree bisection-reconnection (TBR) algorithm, branches collapsing if the maximum branch length was zero. Alignment gaps were treated as missing characters in the analysis of the combined data set, where they occurred in relatively conserved regions. Trees were inferred using the heuristic search option with 1000 random sequence additions, with maxtrees set at 5000. Descriptive tree statistics for parsimony; tree length (TL), consistency index (CI), retention index (RI), relative consistency index (RC) and homoplasy index (HI) were calculated for trees generated under different optimality criteria. The Kishino-Hasegawa tests (Kishino \& Hasegawa 1989) were performed in order to determine whether trees were significantly different. Other details pertaining to analyses (e.g. consideration of TT ratios, comparison of tree topologies and selection of outgroups) are outlined in Jeewon et al. (2003a, b, 2004, 2013).

Bayesian (BI) analyses were performed on MrBayes v. 3.2.1 (Ronquist et al. 2012) based on the models selected by the MrModeltest. The Markov Chain Monte Carlo (MCMC) algorithm of six chains was initiated for $5 \mathrm{M}$ generations in parallel from a random tree topology. The trees were sampled every 200th generation. The distribution of log-likelihood scores was examined to determine the stationary phase for each search and to decide if extra runs were required to achieve convergence, using the program Tracer v. 1.5 (Rambaut \& Drummond 2007). All sampled topologies beneath the asymptote $(10 \%)$ were discarded as part of a burn-in procedure; the remaining trees were used for calculating $\mathrm{PP}$ in the majority rule consensus tree. Posterior probabilities values of the BI analyses (BYPP) over 0.95 were considered significant. 
The ML analyses were conducted with RAxML-HPC BlackBox (v. 8.2.8) (Stamatakis et al. 2008, Stamatakis 2014) in the CIPRES Science Gateway platform (Miller et al. 2010) using a GTR $+\mathrm{I}+\mathrm{G}$ substitution model with 1000 bootstrap replicates. The robustness of the analyses was evaluated by bootstrap support (MLBS).

Phylograms were visualized with FigTree v1.4.0 program (Rambaut 2012) and reorganized in Microsoft power point (2007) and Adobe Illustrator ${ }^{\circledR}$ CS5 (Version 15.0.0, Adobe ${ }^{\circ}$, San Jose, CA).

One hundred and twenty-six taxa are used (including our newly generated sequences) as ingroup taxa, Leptosphaeria conoidea (CBS 616.75) and L. doliolum (CBS 505.75) were selected as outgroup taxa. Sequences generated in this study were deposited in GenBank (Table 1), the final matrices and trees in TreeBASE (accession number: 22328), (Study Accession URL: http://purl.org/phylo/treebase/phylows/study/TB2:S22328) and novel taxonomic descriptions and nomenclature in Faces of Fungi and Index Fungorum as outlined in Jayasiri et al. (2015), Index Fungorum (2018). New species were established based on recommendations outlined by Jeewon \& Hyde (2016).

Table 1 Taxa used in the phylogenetic analysis and their corresponding GenBank numbers. The newly generated sequences are indicated in bold.

\begin{tabular}{|c|c|c|c|c|c|c|}
\hline \multirow{2}{*}{ Species } & \multirow{2}{*}{ Strain no ${ }^{1}$} & \multirow{2}{*}{ Status $^{2}$} & \multicolumn{4}{|c|}{ GenBank Accession no $^{3}$} \\
\hline & & & LSU & ITS & RPB2 & TUB \\
\hline Allophoma minor & CBS 325.82 & $\mathrm{~T}$ & GU238107 & GU237831 & KT389553 & GU237632 \\
\hline Allophoma nicaraguensis & CBS 506.91 & $\mathrm{~T}$ & GU238058 & GU237876 & KT389551 & GU237596 \\
\hline Allophoma piperis & CBS 268.93 & $\mathrm{~T}$ & GU238129 & GU237816 & KT389554 & GU237644 \\
\hline Allophoma tropica & CBS 436.75 & $\mathrm{~T}$ & GU238149 & GU237864 & KT389556 & GU237663 \\
\hline Ascochyta boeremae & CBS 372.84 & $\mathrm{~T}$ & KT389697 & KT389480 & & KT389774 \\
\hline Ascochyta boeremae & CBS 373.84 & & KT389698 & KT389481 & KT389560 & КT389775 \\
\hline $\begin{array}{l}\text { Ascochyta coronillae- } \\
\text { emeri }\end{array}$ & MFLUCC 13-0820 & $\mathbf{T}$ & MH069661 & MH069667 & MH069679 & МH069686 \\
\hline Ascochyta herbicola & CBS 629.97 & $\mathrm{R}$ & GU238083 & GU237898 & KP330421 & GU237614 \\
\hline $\begin{array}{l}\text { Ascochyta medicaginicola } \\
\text { var. macrospora }\end{array}$ & CBS 112.53 & $\mathrm{~T}$ & GU238101 & GU237749 & & GU237628 \\
\hline $\begin{array}{l}\text { Ascochyta medicaginicola } \\
\text { var. macrospora }\end{array}$ & BRIP 45051 & & KY742198 & KY742044 & KY742132 & KY742286 \\
\hline $\begin{array}{l}\text { Ascochyta medicaginicola } \\
\text { var. medicaginicola }\end{array}$ & MFLUCC 16-0599 & & KX698025 & KX698036 & KX698033 & KX698029 \\
\hline Ascochyta phacae & CBS 184.55 & $\mathrm{~T}$ & KT389692 & KT389475 & & KT389769 \\
\hline Ascochyta pisi & CBS 122751 & & KP330444 & KP330432 & EU874867 & KP330388 \\
\hline Ascochyta rabiei & CBS 206.30 & & KT389695 & KT389478 & KT389559 & KT389772 \\
\hline Ascochyta rabiei & CBS 237.37 & $\mathrm{~T}$ & KT389696 & KT389479 & & KT389773 \\
\hline Ascochyta rabiei & CBS 534.65 & & GU237970 & GU237886 & KP330405 & GU237533 \\
\hline $\begin{array}{l}\text { Boeremia exigua var. } \\
\text { heteromorpha }\end{array}$ & CBS 443.94 & $\mathrm{~T}$ & GU237935 & GU237866 & KT389573 & GU237497 \\
\hline $\begin{array}{l}\text { Boeremia exigua var. } \\
\text { opuli }\end{array}$ & CGMCC 3.18354 & $\mathrm{~T}$ & KY742199 & KY742045 & KY742133 & KY742287 \\
\hline Boeremia hedericola & CBS 367.91 & $\mathrm{R}$ & GU237949 & GU237842 & KT389579 & GU237511 \\
\hline Boeremia hedericola & CBS 367.91 & $\mathrm{R}$ & GU237949 & GU237842 & KT389579 & GU237511 \\
\hline $\begin{array}{l}\text { Briansuttonomyces } \\
\text { eucalypti }\end{array}$ & CBS 114879 & $\mathrm{~T}$ & KU728519 & KU728479 & & KU728595 \\
\hline $\begin{array}{l}\text { Briansuttonomyces } \\
\text { eucalypti }\end{array}$ & CBS 114887 & & KU728520 & KU728480 & & KU728596 \\
\hline Calophoma aquilegiicola & CBS 107.96 & $\mathrm{R}$ & GU238041 & GU237735 & KT389586 & GU237581 \\
\hline Calophoma clematidina & CBS 102.66 & & FJ515630 & FJ426988 & KT389587 & FJ427099 \\
\hline Calophoma clematidina & CBS 108.79 & $\mathrm{~T}$ & FJ515632 & FJ426989 & KT389588 & FJ427100 \\
\hline
\end{tabular}


Table 1 Continued.

\begin{tabular}{|c|c|c|c|c|c|c|}
\hline \multirow{2}{*}{ Species } & \multirow{2}{*}{ Strain no ${ }^{1}$} & \multirow{2}{*}{ Status $^{2}$} & \multicolumn{4}{|c|}{ GenBank Accession no ${ }^{3}$} \\
\hline & & & LSU & ITS & RPB2 & TUB \\
\hline Calophoma rosae & CGMCC 3.18347 & $\mathrm{~T}$ & KY742203 & KY742049 & KY742135 & KY742291 \\
\hline Cumuliphoma indica & CBS 654.77 & $\mathrm{~T}$ & GU238122 & FJ427043 & LT623261 & FJ427153 \\
\hline Cumuliphoma omnivirens & CBS 341.86 & $\mathrm{~T}$ & LT623214 & FJ427042 & LT623260 & FJ427152 \\
\hline Cumuliphoma pneumoniae & CBS 142454 & $\mathrm{~T}$ & LN907392 & LT592925 & LT593063 & LT592994 \\
\hline Didymella aquatica & CGMCC 3.18349 & $\mathrm{~T}$ & KY742209 & KY742055 & KY742140 & KY742297 \\
\hline Didymella arachidicola & CBS 333.75 & $\mathrm{~T}$ & GU237996 & GU237833 & KT389598 & GU237554 \\
\hline Didymella exigua & CBS 183.55 & $\mathrm{~T}$ & EU754155 & GU237794 & EU874850 & GU237525 \\
\hline Didymella heteroderae & CBS 109.92 & $\mathrm{~T}$ & GU238002 & FJ426983 & KT389601 & FJ427098 \\
\hline Didymella macrophylla & CGMCC 3.18357 & $\mathrm{~T}$ & KY742224 & KY742070 & KY742154 & KY742312 \\
\hline $\begin{array}{l}\text { Didymellocamarosporium } \\
\text { tamaricis }\end{array}$ & MFLUCC 14-0241 & $\mathrm{T}$ & KU848183 & & & \\
\hline Didysimulans italica & MFLUCC 15-0059 & $\mathrm{T}$ & KY496730 & KY496750 & KY514408 & \\
\hline Didysimulans mezzanensis & MFLUCC 15-0067 & $\mathrm{T}$ & KY496733 & KY496753 & KY514411 & \\
\hline Ectophoma multirostrata & CBS 274.60 & $\mathrm{~T}$ & GU238111 & FJ427031 & LT623265 & FJ427141 \\
\hline Ectophoma multirostrata & CBS 368.65 & & GU238112 & FJ427033 & LT623266 & FJ427143 \\
\hline Ectophoma pomi & CBS 267.92 & $\mathrm{~T}$ & GU238128 & GU237814 & LT623263 & GU237643 \\
\hline Endocoryneum festucae & MFLUCC 14-0461 & $\mathrm{T}$ & KU848203 & & & \\
\hline Epicoccum brasiliense & CBS 120105 & $\mathrm{~T}$ & GU238049 & GU237760 & KT389627 & GU237588 \\
\hline Epicoccum camelliae & CGMCC 3.18343 & $\mathrm{~T}$ & KY742245 & KY742091 & KY742170 & KY742333 \\
\hline Epicoccum huancayense & CBS 105.80 & $\mathrm{~T}$ & GU238084 & GU237732 & КT389630 & GU237615 \\
\hline Epicoccum latusicollum & CGMCC 3.18346 & $\mathrm{~T}$ & KY742255 & KY742101 & KY742174 & KY742343 \\
\hline Epicoccum nigrum & CBS 173.73 & $\mathrm{~T}$ & GU237975 & FJ426996 & КT389632 & FJ427107 \\
\hline $\begin{array}{l}\text { Heterophoma } \\
\text { verbascicola }\end{array}$ & CGMCC 3.18364 & $\mathrm{~T}$ & KY742273 & KY742119 & KY742187 & KY742361 \\
\hline $\begin{array}{l}\text { Heterophoma } \\
\text { verbascicola }\end{array}$ & LC 8164 & & KY742274 & KY742120 & KY742188 & KY742362 \\
\hline Heterophoma adonidis & CBS 114309 & & KT389724 & KT389506 & KT389637 & KT389803 \\
\hline $\begin{array}{l}\text { Heterophoma } \\
\text { dictamnicola }\end{array}$ & CBS 507.91 & & GU238065 & GU237877 & KT389638 & GU237603 \\
\hline Juxtiphoma eupyrena & CBS 374.91 & & GU238072 & FJ426999 & LT623268 & FJ427110 \\
\hline Juxtiphoma eupyrena & CBS 527.66 & & GU238073 & FJ427000 & LT623269 & FJ427111 \\
\hline Leptosphaeria conoidea & CBS 616.75 & & JF740279 & JF740201 & KT389639 & KT389804 \\
\hline Leptosphaeria doliolum & CBS 505.75 & $\mathrm{~T}$ & GQ387576 & JF740205 & KT389640 & JF740144 \\
\hline $\begin{array}{l}\text { Leptosphaerulina } \\
\text { americana }\end{array}$ & CBS 213.55 & & GU237981 & GU237799 & KT389641 & GU237539 \\
\hline $\begin{array}{l}\text { Leptosphaerulina } \\
\text { arachidicola }\end{array}$ & CBS 275.59 & & GU237983 & GU237820 & & GU237543 \\
\hline Leptosphaerulina australis & CBS 317.83 & & EU754166 & GU237829 & GU371790 & GU237540 \\
\hline Leptosphaerulina trifolii & CBS 235.58 & & GU237982 & GU237806 & & GU237542 \\
\hline $\begin{array}{l}\text { Macroventuria } \\
\text { anomochaeta }\end{array}$ & CBS 502.72 & & GU237985 & GU237873 & & GU237545 \\
\hline $\begin{array}{l}\text { Macroventuria } \\
\text { anomochaeta }\end{array}$ & CBS 525.71 & $\mathrm{~T}$ & GU237984 & GU237881 & GU456346 & GU237544 \\
\hline Macroventuria wentii & CBS 526.71 & $\mathrm{~T}$ & GU237986 & GU237884 & KT389642 & GU237546 \\
\hline $\begin{array}{l}\text { Microsphaeropsis } \\
\text { olivacea }\end{array}$ & CBS 442.83 & & EU754171 & GU237865 & & GU237547 \\
\hline $\begin{array}{l}\text { Microsphaeropsis } \\
\text { olivacea }\end{array}$ & CBS 233.77 & & GU237988 & GU237803 & KT389643 & GU237549 \\
\hline
\end{tabular}


Table 1 Continued.

\begin{tabular}{|c|c|c|c|c|c|c|}
\hline \multirow{2}{*}{ Species } & \multirow{2}{*}{ Strain no ${ }^{1}$} & \multirow{2}{*}{ Status $^{2}$} & \multicolumn{4}{|c|}{ GenBank Accession no ${ }^{3}$} \\
\hline & & & LSU & ITS & RPB2 & TUB \\
\hline $\begin{array}{l}\text { Microsphaeropsis } \\
\text { olivacea }\end{array}$ & CBS 432.71 & & GU237987 & GU237863 & & GU237548 \\
\hline $\begin{array}{l}\text { Microsphaeropsis } \\
\text { olivacea }\end{array}$ & MFLUCC 14-0507 & & KR025863 & KR025859 & & \\
\hline Microsphaeropsis proteae & CPC 1425 & & JN712563 & JN712497 & & JN712650 \\
\hline Microsphaeropsis proteae & СРC 1424 & & JN712562 & JN712496 & & JN712649 \\
\hline Microsphaeropsis proteae & CPC 1423 & & JN712561 & JN712495 & & \\
\hline $\begin{array}{l}\text { Microsphaeropsis spartii- } \\
\text { juncei }\end{array}$ & MFLU 16-0100 & $\mathbf{T}$ & МH069663 & МH069669 & МH069681 & МH069688 \\
\hline $\begin{array}{l}\text { Microsphaeropsis spartii- } \\
\text { juncei }\end{array}$ & MFLU 16-0097 & & MH069662 & MH069668 & MH069680 & МH069687 \\
\hline Neoascochyta desmazieri & CBS 297.69 & $\mathrm{~T}$ & KT389726 & KT389508 & KT389644 & KT389806 \\
\hline Neoascochyta europaea & CBS 820.84 & $\mathrm{~T}$ & KT389729 & KT389511 & KT389646 & KT389809 \\
\hline Neoascochyta paspali & CBS 560.81 & $\mathrm{~T}$ & GU238124 & FJ427048 & KP330426 & FJ427158 \\
\hline Neoascochyta triticicola & CBS 544.74 & $\mathrm{~T}$ & EU754134 & GU237887 & KT389652 & GU237488 \\
\hline $\begin{array}{l}\text { Neodidymella } \\
\text { thailandicum }\end{array}$ & MFLUCC 11-0140 & $\mathrm{T}$ & MG520976 & MG520956 & & \\
\hline $\begin{array}{l}\text { Neodidymelliopsis } \\
\text { achlydis }\end{array}$ & CBS 256.77 & $\mathrm{~T}$ & KT389749 & KT389531 & & KT389829 \\
\hline $\begin{array}{l}\text { Neodidymelliopsis } \\
\text { cannabis }\end{array}$ & CBS 234.37 & & GU237961 & GU237804 & KP330403 & GU237523 \\
\hline $\begin{array}{l}\text { Neodidymelliopsis } \\
\text { polemonii }\end{array}$ & CBS 109181 & $\mathrm{~T}$ & GU238133 & GU237746 & KP330427 & GU237648 \\
\hline $\begin{array}{l}\text { Neodidymelliopsis } \\
\text { xanthina }\end{array}$ & CBS 383.68 & $\mathrm{~T}$ & GU238157 & GU237855 & KP330431 & GU237668 \\
\hline $\begin{array}{l}\text { Neomicrosphaeropsis } \\
\text { alhagi-pseudalhagi }\end{array}$ & MFLUCC 17-0825 & $\mathbf{T}$ & MH069664 & MH069670 & MH069682 & MH069689 \\
\hline $\begin{array}{l}\text { Neomicrosphaeropsis } \\
\text { cytisi }\end{array}$ & MFLUCC 13-0396 & & KX572342 & KX572337 & KX572355 & \\
\hline $\begin{array}{l}\text { Neomicrosphaeropsis } \\
\text { cytisicola }\end{array}$ & MFLU 16-0114 & $\mathbf{T}$ & MH069665 & MH069671 & MH069683 & МH069690 \\
\hline $\begin{array}{l}\text { Neomicrosphaeropsis } \\
\text { cytisinus }\end{array}$ & MFLUCC 16-0790 & $\mathrm{T}$ & KX611241 & & & \\
\hline $\begin{array}{l}\text { Neomicrosphaeropsis } \\
\text { elaeagni }\end{array}$ & MFLUCC 17-0740 & $\mathbf{T}$ & MH069666 & MH069672 & MH069684 & MH069691 \\
\hline $\begin{array}{l}\text { Neomicrosphaeropsis } \\
\text { italica }\end{array}$ & MFLUCC 15-0485 & $\mathrm{T}$ & KU729854 & KU900318 & KU674820 & \\
\hline $\begin{array}{l}\text { Neomicrosphaeropsis } \\
\text { italica }\end{array}$ & MFLUCC 15-0484 & & KU729853 & KU900319 & KU695539 & KX453298 \\
\hline $\begin{array}{l}\text { Neomicrosphaeropsis } \\
\text { italica }\end{array}$ & MFLUCC 16-0284 & & KU900296 & KU900321 & & KX453299 \\
\hline $\begin{array}{l}\text { Neomicrosphaeropsis } \\
\text { minima }\end{array}$ & MFLUCC 13-0394 & & KX572341 & KX572336 & & \\
\hline $\begin{array}{l}\text { Neomicrosphaeropsis } \\
\text { novorossica }\end{array}$ & MFLUCC 14-0578 & $\mathrm{T}$ & KX198710 & KX198709 & & \\
\hline $\begin{array}{l}\text { Neomicrosphaeropsis } \\
\text { rossica }\end{array}$ & MFLUCC 14-0586 & $\mathrm{T}$ & KU729855 & KU752192 & & \\
\hline $\begin{array}{l}\text { Neomicrosphaeropsis } \\
\text { tamaricicola }\end{array}$ & MFLUCC 14-0443 & & KU729851 & KU900322 & & \\
\hline $\begin{array}{l}\text { Neomicrosphaeropsis } \\
\text { tamaricicola }\end{array}$ & MFLUCC 14-0439 & & KU729858 & KU900323 & & \\
\hline $\begin{array}{l}\text { Neomicrosphaeropsis } \\
\text { tamaricicola }\end{array}$ & MFLUCC 14-0602 & $\mathrm{T}$ & KM408754 & KM408753 & MH069684 & MH069691 \\
\hline Nothophoma anigozanthi & CBS 381.91 & $\mathrm{~T}$ & GU238039 & GU237852 & KT389655 & GU237580 \\
\hline
\end{tabular}


Table 1 Continued.

\begin{tabular}{|c|c|c|c|c|c|c|}
\hline \multirow{2}{*}{ Species } & \multirow{2}{*}{ Strain no ${ }^{1}$} & \multirow{2}{*}{ Status $^{2}$} & \multicolumn{4}{|c|}{ GenBank Accession no ${ }^{3}$} \\
\hline & & & LSU & ITS & RPB2 & TUB \\
\hline $\begin{array}{l}\text { Nothophoma arachidis- } \\
\text { hypogaeae }\end{array}$ & CBS 125.93 & $\mathrm{R}$ & GU238043 & GU237771 & KT389656 & GU237583 \\
\hline Nothophoma gossypiicola & CBS 377.67 & & GU238079 & GU237845 & KT389658 & GU237611 \\
\hline Nothophoma infossa & CBS 123395 & $\mathrm{~T}$ & GU238089 & FJ427025 & KT389659 & FJ427135 \\
\hline Nothophoma quercina & CBS 633.92 & & EU754127 & GU237900 & KT389657 & GU237609 \\
\hline Paraboeremia adianticola & CBS 187.83 & & GU238035 & GU237796 & KP330401 & GU237576 \\
\hline Paraboeremia camellae & CGMCC 3.18106 & $\mathrm{~T}$ & KX829042 & KX829034 & KX829050 & KX829058 \\
\hline Paraboeremia litseae & CGMCC 3.18109 & $\mathrm{~T}$ & KX829037 & KX829029 & KX829045 & KX829053 \\
\hline $\begin{array}{l}\text { Paraboeremia } \\
\text { oligotrophica }\end{array}$ & CGMCC 3.18111 & $\mathrm{~T}$ & KX829039 & KX829031 & KX829047 & KX829055 \\
\hline $\begin{array}{l}\text { Paraboeremia } \\
\text { selaginellae }\end{array}$ & CBS 122.93 & $\mathrm{~T}$ & GU238142 & GU237762 & & GU237656 \\
\hline Phoma herbarum & CBS 134.96 & & KT389753 & KT389535 & KT389661 & KT389834 \\
\hline Phoma herbarum & CBS 274.37 & & KT389754 & KT389537 & KT389662 & KT389835 \\
\hline Phoma herbarum & CBS 377.92 & & KT389756 & KT389536 & KT389663 & KT389837 \\
\hline Phoma herbarum & CBS 502.91 & & GU238082 & GU237874 & KP330419 & GU237613 \\
\hline Phoma herbarum & CBS 615.75 & $\mathrm{R}$ & EU754186 & FJ427022 & KP330420 & FJ427133 \\
\hline Phomatodes aubrietiae & CBS 383.67 & $\mathrm{R}$ & GU238044 & GU237854 & & GU237584 \\
\hline Phomatodes aubrietiae & CBS 627.97 & $\mathrm{~T}$ & GU238045 & GU237895 & KT389665 & GU237585 \\
\hline Phomatodes nebulosa & CBS 117.93 & & GU238114 & GU237757 & KP330425 & GU237633 \\
\hline Phomatodes nebulosa & CBS 740.96 & & KT389758 & KT389540 & KT389667 & KT389839 \\
\hline Phomatodes nebulosa & CBS 100191 & & KP330446 & KP330434 & KT389666 & KP330390 \\
\hline $\begin{array}{l}\text { Pseudoascochyta novae- } \\
\text { zelandiae }\end{array}$ & CBS 141689 & & LT592893 & LT592892 & LT592895 & LT592894 \\
\hline $\begin{array}{l}\text { Pseudohendersonia } \\
\text { galiorum }\end{array}$ & $\begin{array}{l}\text { MFLUCC } 14- \\
0452\end{array}$ & $\mathrm{~T}$ & KU848207 & & & \\
\hline $\begin{array}{l}\text { Remotididymella } \\
\text { anthropophila }\end{array}$ & CBS 142462 & $\mathrm{~T}$ & LN907421 & LT592936 & LT593075 & LT593005 \\
\hline $\begin{array}{l}\text { Remotididymella } \\
\text { destructiva }\end{array}$ & CBS 133.93 & & GU238064 & GU237779 & LT623257 & GU237602 \\
\hline $\begin{array}{l}\text { Remotididymella } \\
\text { destructiva }\end{array}$ & CBS 378.73 & $\mathrm{~T}$ & GU238063 & GU237849 & LT623258 & GU237601 \\
\hline Similiphoma crystallifera & CBS 193.82 & $\mathrm{~T}$ & GU238060 & GU237797 & LT623267 & GU237598 \\
\hline Stagonosporopsis actaeae & CBS 106.96 & $\mathrm{~T}$ & GU238166 & GU237734 & KT389672 & GU237671 \\
\hline $\begin{array}{l}\text { Stagonosporopsis } \\
\text { crystalliniformis }\end{array}$ & CBS 713.85 & $\mathrm{~T}$ & GU238178 & GU237903 & KT389675 & GU237683 \\
\hline Stagonosporopsis dennisii & CBS 631.68 & $\mathrm{~T}$ & GU238182 & GU237899 & KT389677 & GU237687 \\
\hline $\begin{array}{l}\text { Stagonosporopsis } \\
\text { helianthi }\end{array}$ & CBS 200.87 & $\mathrm{~T}$ & KT389761 & KT389545 & KT389683 & KT389848 \\
\hline Vacuiphoma bulgarica & CBS 357.84 & $\mathrm{~T}$ & GU238050 & GU237837 & LT623256 & GU237589 \\
\hline Vacuiphoma oculihominis & UTHSC DI16-308 & $\mathrm{T}$ & LN907451 & LT592954 & LT593093 & LT593023 \\
\hline Xenodidymella applanata & CBS 195.36 & $\mathrm{~T}$ & KT389764 & KT389548 & & KT389852 \\
\hline Xenodidymella applanata & CBS 115577 & & KT389762 & KT389546 & KT389688 & KT389850 \\
\hline Xenodidymella catariae & CBS 102635 & & GU237962 & GU237727 & KP330404 & GU237524 \\
\hline
\end{tabular}

1 BRIP: Plant Pathology Herbarium, Department of Employment, Economic, Development and Innovation, Queensland, Australia; CBS: Westerdijk Fungal Biodiversity Institute (formerly CBSKNAW), Utrecht, The Netherlands; CGMCC: China General Microbiological Culture Collection, Beijing, China; CPC: Culture collection of Pedro Crous, housed at CBS; LC: Corresponding author's personal collection deposited in laboratory, housed at CAS, China; MFLUCC: Mae Fah Luang University Culture Collection, Chiang Rai, Thailand; UTHSC, Fungus Testing Laboratory at the University of Texas Health Science Center, San Antonio, Texas, USA. 
${ }^{2}$ T: ex-type strain; R: representative strain.

${ }^{3}$ ITS: internal transcibed spacer regions $1 \& 2$ including 5.8S nrDNA gene; LSU: 28S large subunit of the nrRNA gene; rpb2: RNA polymerase II second subunit; tub2: ß-tubulin.

\section{Results and Discussion}

\section{Phylogenetic analyses}

Topologies of trees (under ML, MP and BI criteria) recovered for each gene dataset were visually compared and the overall tree topology was congruent to those obtained from the combined dataset.

The RAxML analysis of the combined dataset yielded a best scoring tree (Fig. 1) with a final ML optimization likelihood value of -23881.01104. The matrix had 734 distinct alignment patterns, with $8.95 \%$ proportion of gaps and completely undetermined characters in this alignment. Parameters for the GTR + I + G model of the combined LSU, ITS, $r p b 2$ and $t u b 2$ were as follows: Estimated base frequencies were as follows: $\mathrm{A}=0.238058, \mathrm{C}=0.241410, \mathrm{G}=0.27525, \mathrm{~T}=$ 0.245283; substitution rates $\mathrm{AC}=1.943648, \mathrm{AG}=6.96474, \mathrm{AT}=2.220889, \mathrm{CG}=0.925886, \mathrm{CT}=$ 14.019529, GT $=1.000$; proportion of invariable sites $\mathrm{I}=0.63074$; gamma distribution shape parameter $\alpha=0.584276$. The maximum parsimonious dataset for the combined gene sequences consisted of 2231 characters, of which 1560 were constant, $615(27.6 \%)$ parsimony-informative and 56 parsimony-uninformative. The parsimony analysis of the data matrix resulted in the maximum of 2325 equally most parsimonious trees with a length of 4662 steps $(\mathrm{CI}=0.238, \mathrm{RI}=$ $0.636, \mathrm{RC}=0.151, \mathrm{HI}=0.762$ ) in the first tree. The Bayesian analysis resulted in 25001 trees after $5 \mathrm{M}$ generations with 0.009735 as the average standard deviation of split frequency. Therefore, the first 2500 trees, representing the burn-in phase of the analyses, were discarded, while the remaining 22501 trees were used or calculating posterior probabilities in the majority rule consensus tree.

Newly generated sequences from two Microsphaeropsis isolates (MFLU 16-0100 and MFLU 16-0097) grouped with isolates currently circumscribed as Microsphaeropsis olivacea and M. proteae (de Gruyter et al. 2009, Aveskamp et al. 2010, Crous et al. 2011, Verkley et al. 2014, Chen et al. 2015). These taxa formed an isolated clade (Clade A, Fig 1) within Didymellaceae, but poorly supported in multi-gene analyses $(69 \%$ in ML, $<60 \%$ in MP and $<0.95$ in BI). Within Clade A (Fig 1), our novel isolates are closely related and monophyletic with Microsphaeropsis olivacea (CBS 442.83, CBS 432.71, CBS 233.77) and retrieved 67\% (ML), 86\% (MP), 1.00 (BI) bootstrap support for this lineage (Subclade A1).

Ascochyta coronillae-emeri (MFLUCC 13-0820), showed a close phylogenetic affinity to $A$. rabiei (CBS 206.30, CBS 237.37, CBS 534.65), A. phacae (CBS 184.55) and A. herbicola (CBS 629.97) in the combined phylogeny (Subclade B1) and this relationship retrieved $96 \%$ ML, 92\% MP and $1.00 \mathrm{BI}$ support.

Three newly generated sequences, Neomicrosphaeropsis alhagi-pseudalhagi (MFLUCC 170825), N. cytisicola (MFLU 16-0114) and N. elaeagni (MFLUCC 17-0740), grouped with Didymellocamarosporium tamaricis and eleven Neomicrosphaeropsis isolates. These taxa form a monophyletic clade (Clade C) in Didymellaceae with poor statistical support (65\% in ML, <60\% in MP and $<0.95$ in BI). Didymellocamarosporium tamaricis, Neomicrosphaeropsis elaeagni sp. nov., $N$. italica, $N$. novorossica, $N$. rossica and $N$. tamaricicola forms a subclade (Subclade C1) in the combined phylogeny with $86 \%$ ML $77 \%$ MP and 1.00 BI support. Neomicrosphaeropsis cytisi, $N$. cytisicola sp. nov., N. cytisinus and $N$. minima forms a separate cluster (Subclade C3) within Clade C with high statistical support (91\% ML, 84\% MP and $1.00 \mathrm{BI})$. Neomicrosphaeropsis alhagi-pseudalhagi sp. nov. nested in between subclades $\mathrm{C} 1$ and $\mathrm{C} 3$. 


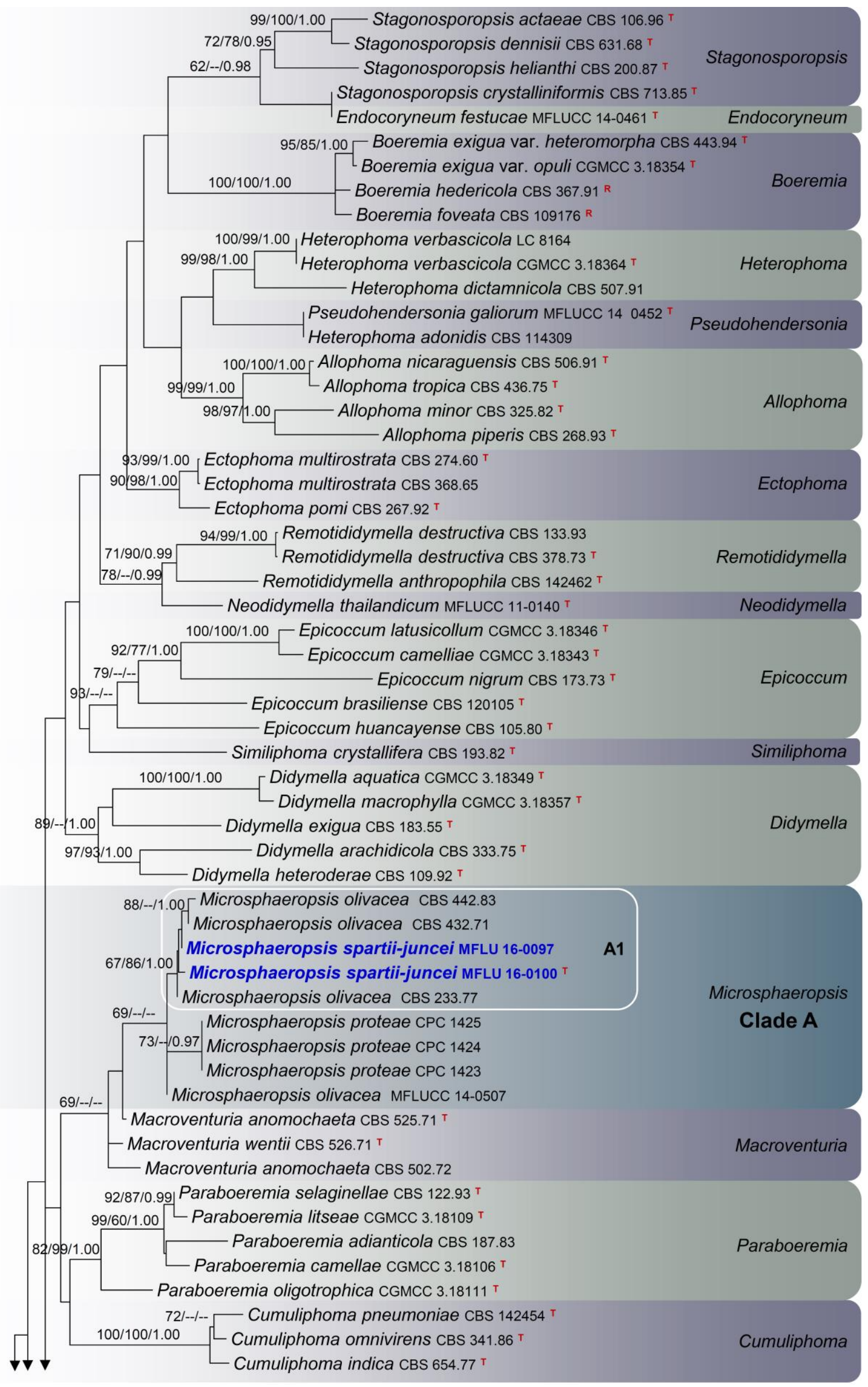




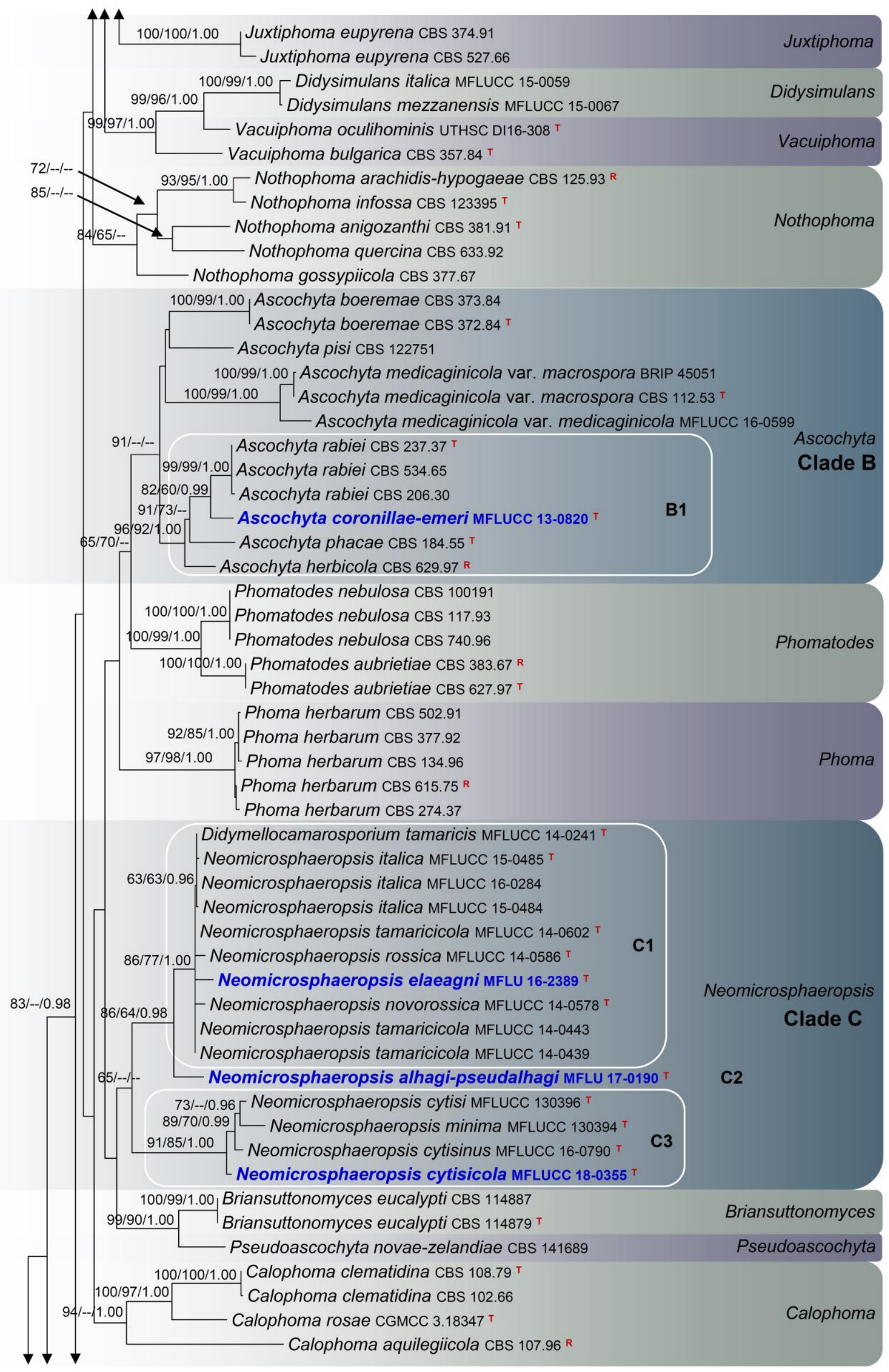




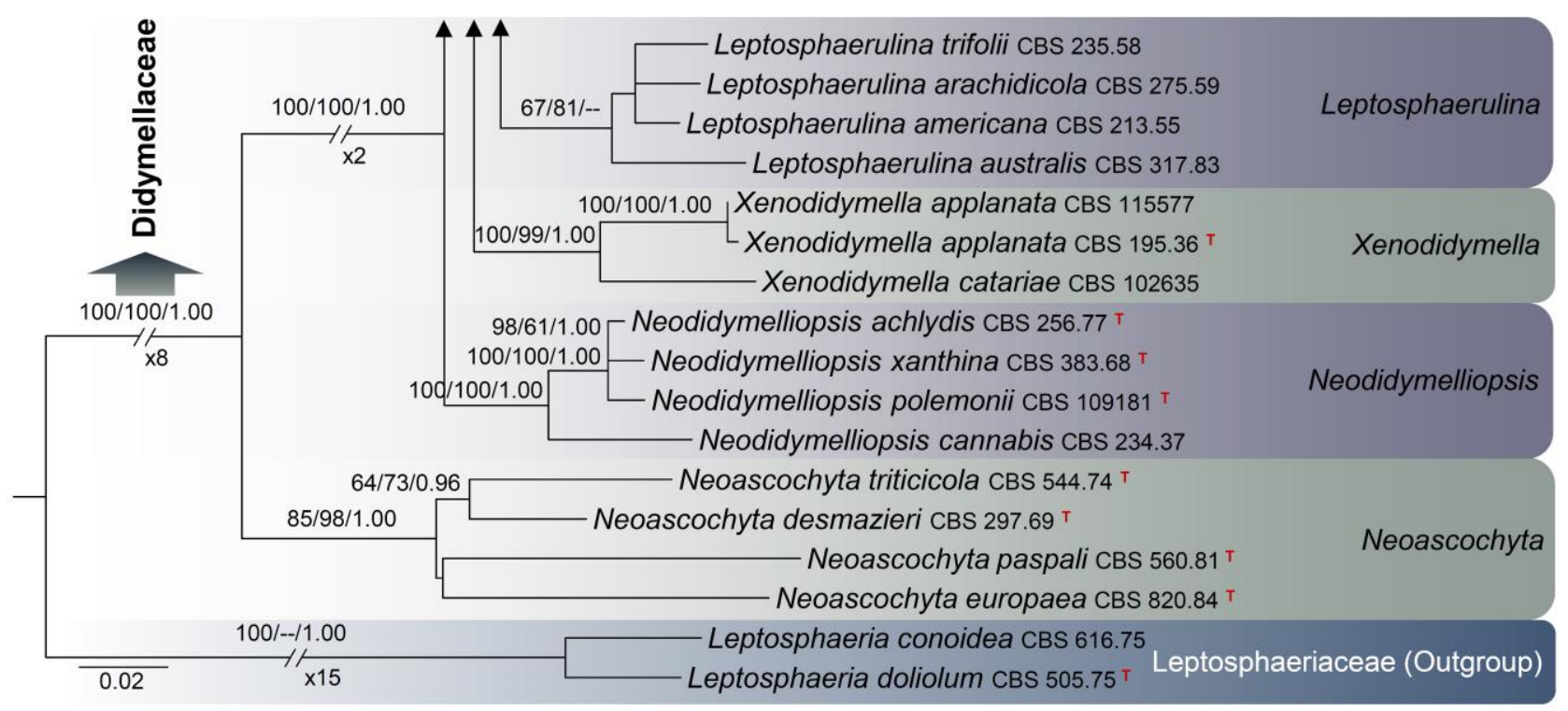

Fig. 1 - RAxML tree based on analysis of a combined dataset of LSU, ITS, rpb2 and tub2 partial sequence data. Bootstrap support values for ML and MP equal to or greater than $60 \%$, Bayesian posterior probabilities (PP) equal to or greater than 0.95 are defined as ML/MP/PP above the nodes. Genera, where known, and selected regions are indicated with coloured blocks. The new isolates are in blue. The ex-type strains are noted with superscripted $\mathrm{T}$ and representative strains are noted with superscripted $\mathrm{R}$. The scale bar represents the expected number of nucleotide substitutions per site.

\section{Taxonomy}

Based on the results of the combined multi-gene phylogenies (Fig. 1), morphological observations, five novel species are described.

Ascochyta coronillae-emeri Wanas., Camporesi, E.B.G. Jones \& K.D. Hyde, sp. nov.

Fig. 3 Index Fungorum number: IF554394; Facesoffungi number: FoF 04466 isolated.

Etymology - Name reflects the host species Coronilla emerus, from which the species was

Holotype - MFLU 16-0163.

Saprobic on Coronilla emerus L. Sexual morph: Ascomata 120-150 $\mu \mathrm{m}$ high, 150-220 $\mu \mathrm{m}$ diam. $(\overline{\mathrm{x}}=133.1 \times 186.1 \mu \mathrm{m}, \mathrm{n}=5)$, immersed to semi-erumpent, globose or subglobose, dark brown to black, coriaceous. Peridium 10-15 $\mu \mathrm{m}$ wide at the base, $15-20 \mu \mathrm{m}$ wide at the sides, comprising reddish to dark brown cells of textura angularis. Hamathecium comprising numerous, 2-3 $\mu \mathrm{m}$ wide, filamentous, branched, septate, pseudoparaphyses. Asci $90-110 \times 25-35 \mu \mathrm{m}(\overline{\mathrm{x}}=$ $100.3 \times 29.1 \mu \mathrm{m}, \mathrm{n}=20$ ), 8 -spored, bitunicate, fissitunicate, clavate, pedicellate, thick-walled at the apex, with minute ocular chamber. Ascospores 36-35 $\times 13-15 \mu \mathrm{m}(\overline{\mathrm{x}}=32.7 \times 13.8 \mu \mathrm{m}, \mathrm{n}=30)$, overlapping biseriate, mostly ellipsoidal, muriform, 4-6-transversely septate, with 1 vertical septum, slightly constricted at the septa, initially hyaline to pale yellow, becoming brown to dark brown at maturity, upper part wider than the lower part, rounded at both end, surrounded by a thick mucilaginous sheath (20-30 $\mu \mathrm{m}$ wide). Asexual morph: coelomycetous. Conidiomata superficial or immersed in the agar, pale brown to dark brown, 0.5-1 mm diam, simple, or complex with several merging cavities. Conidiomatal wall composed of textura angularis cells. Conidiogenous cells discrete, assembled into protruding masses of cells, or integrated in very compact conidiophores. Conidia 6-7 × 1.9-2.4 $\mu \mathrm{m}(\overline{\mathrm{x}}=6.3 \times 2.1 \mu \mathrm{m}, \mathrm{n}=30)$, ellipsoidal or short-cylindrical, hyaline, straight or slightly curved, rounded at both ends, 1-celled, with 1-2 small, guttules.

Known distribution - On Coronilla emerus, Italy. 


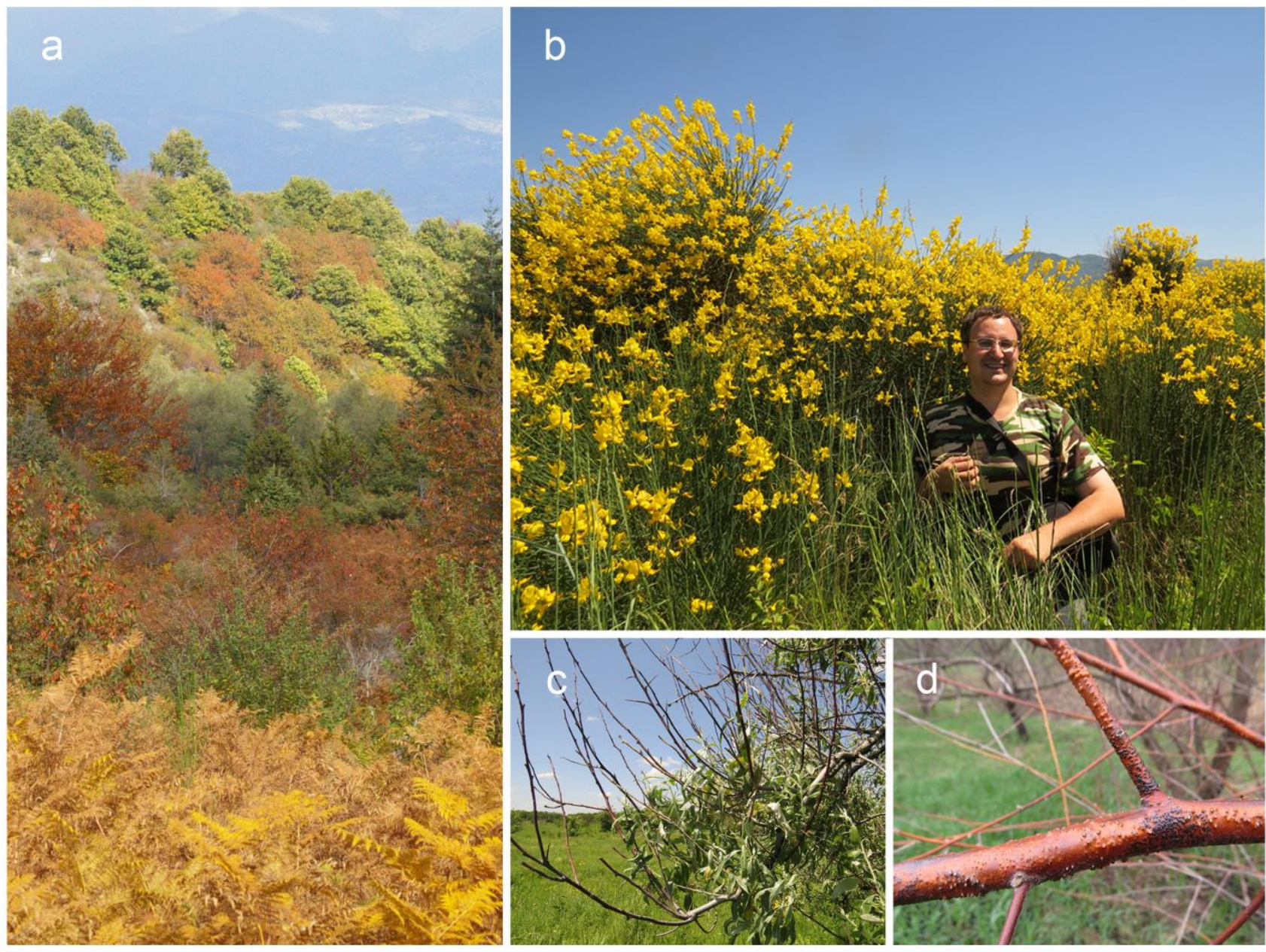

e

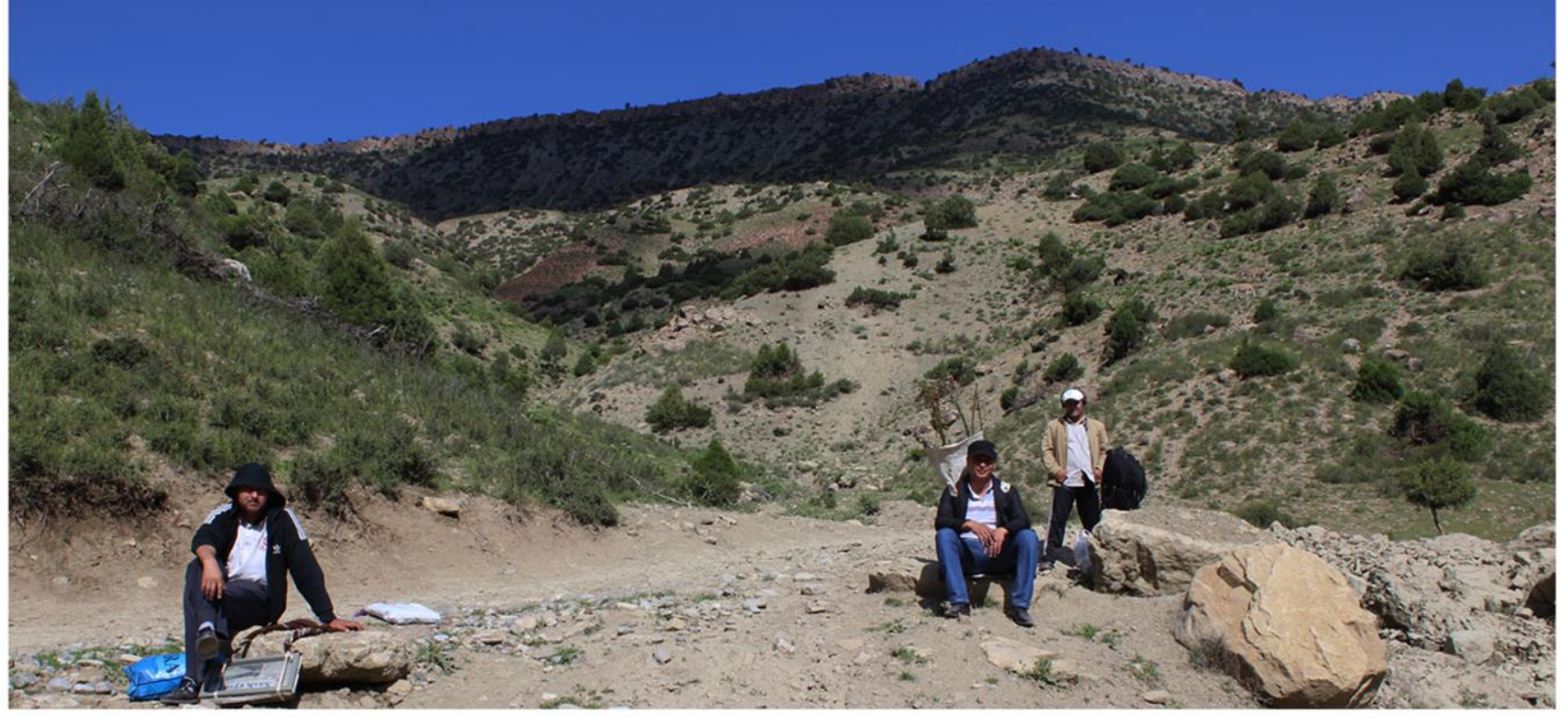

Fig. 2 - Habitats. a. Italy (Bagno di Cetica). b-d Russia (c, d Elaeagnus angustifolia L.). e Uzbekistan. Photos by Erio Camporesi, Timur Bulgakov and Yusufjon Gafforov. 


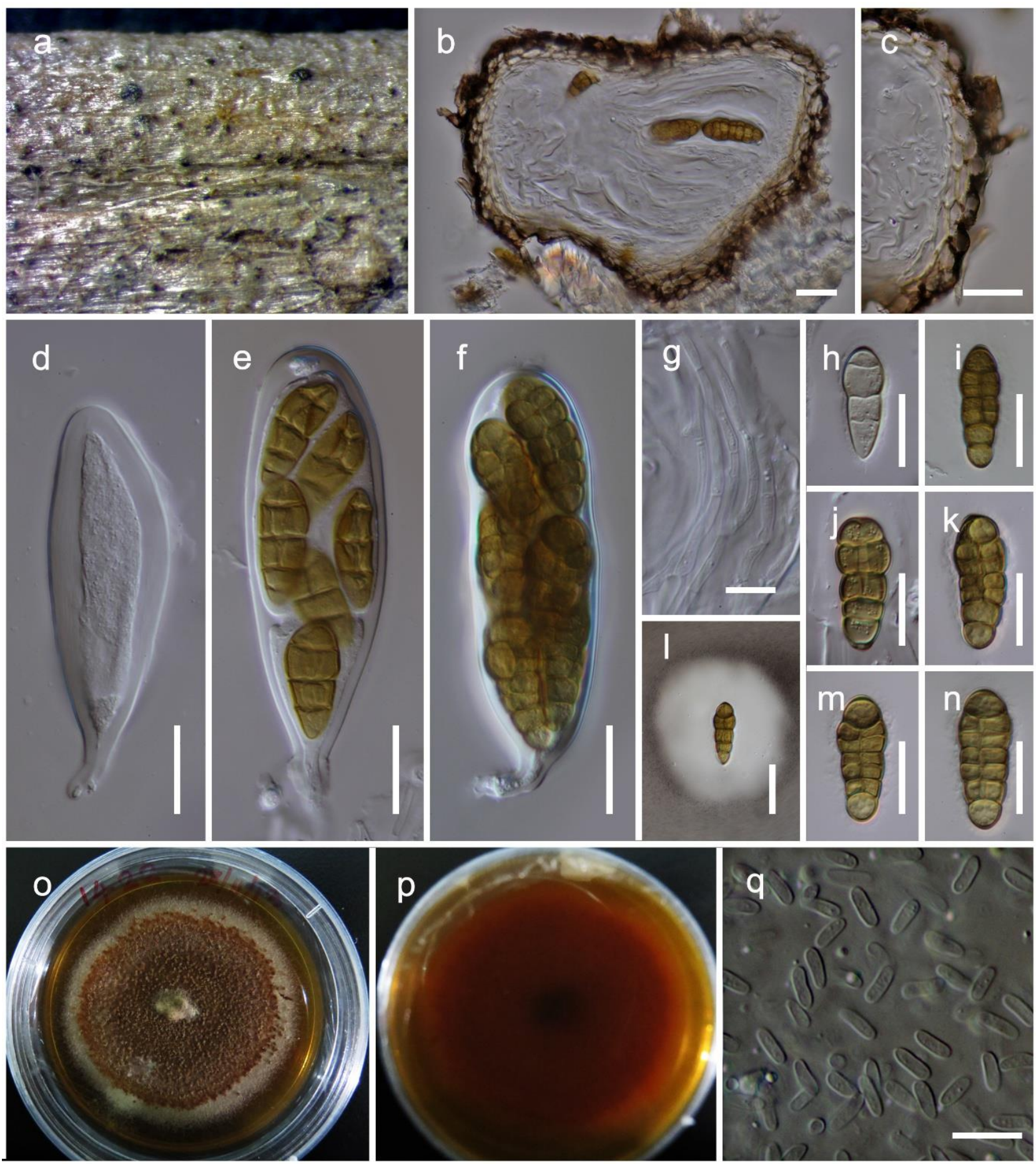

Fig. 3 - Ascochyta coronillae-emeri (MFLU 16-0163, holotype). a Appearance of ascomata on host substrate. b Section of ascoma. c Peridium. d-f Asci. g Pseudoparaphyses. i-n Ascospores (Note the ascospore stained with Indian Ink in 1). o, p Culture on PDA (note p reverse). q Conidia. Scale bars: b-f, h-n $=20 \mu \mathrm{m}, \mathrm{g}, \mathrm{q}=10 \mu \mathrm{m}$.

Material examined - ITALY, Forlì-Cesena Province, Bagno di Romagna, Valbonella, on dead aerial branch of Coronilla emerus (Fabaceae) 23 August 2013, E. Camporesi IT 1422 (MFLU 16-0163, holotype); ex-type living culture, MFLUCC 13-0820.

Notes - Muriform ascospores are reported here for the first time in this genus. The new fungus was collected from Coronilla emerus in Italy and it morphologically resembles most of the Pleosporaceae taxa (e.g. Alternaria, Comoclathris, Pleospora) by its clavate, pedicellate asci with thick-walled at the apex and mostly ellipsoidal, muriform, brown ascospores. However, 
phylogenetically it has a close affinity to Ascochyta herbicola, A. phacae and A. rabiei in Didymellaceae (subclade B1, Fig. 1). Among them, the sexual morph is known only for Ascochyta phacae, which differs from our new isolate in having cylindrical to subclavate asci and hyaline, uniseptate ascospores. Though the ascospore characters are different of our new isolate from all other Ascochyta species, its thin peridium and asexual morph characteristics (ellipsoidal or shortcylindrical, hyaline conidia) are in agreement with its phylogenetic placement within Ascochyta.

Microsphaeropsis spartii-juncei Wanas., Camporesi, E.B.G. Jones \& K.D. Hyde, sp. nov. $\quad$ Fig. 4 Index Fungorum number: IF554395; Facesoffungi number: FoF 04467

Etymology - Name reflects the host species Spartium junceum, from which the species was isolated.

Holotype - MFLU 16-0100.

Saprobic on Spartium junceum L. Sexual morph: Ascomata 180-250 $\mu \mathrm{m}$ high, 180-220 $\mu \mathrm{m}$ diam. $(\overline{\mathrm{x}}=219.7 \times 206.9 \mu \mathrm{m}, \mathrm{n}=5)$, immersed to semi-erumpent, globose or subglobose, dark brown to black, coriaceous. Peridium 10-15 $\mu \mathrm{m}$ wide at the base, 15-30 $\mu \mathrm{m}$ wide at the sides, comprising reddish to dark brown cells of textura angularis. Hamathecium comprising numerous, 2-3 $\mu \mathrm{m}$ wide, filamentous, branched, septate, pseudoparaphyses. Asci $120-140 \times 28-35 \mu \mathrm{m}(\overline{\mathrm{x}}=$ $133.4 \times 31.3 \mu \mathrm{m}, \mathrm{n}=20$ ), 8 -spored, bitunicate, fissitunicate, clavate, pedicellate, thick-walled at the apex, with minute ocular chamber. Ascospores 32-36 $\times 13-15 \mu \mathrm{m}(\overline{\mathrm{x}}=34.7 \times 13.7 \mu \mathrm{m}, \mathrm{n}=30)$, overlapping biseriate, mostly ellipsoidal, muriform, 6-7-transversely septate, with 1-2 vertical septa, slightly constricted at the septa, initially hyaline to pale yellow, becoming brown to dark brown at maturity, rounded at both end, surrounded by a thick mucilaginous sheath $(15-20 \mu \mathrm{m}$ wide). Asexual morph: coelomycetous. Conidiomata superficial or immersed in the agar, pale brown to dark brown, 0.5-1 $\mathrm{mm}$ diam, simple, or complex with several merging cavities. Conidiomatal wall composed of textura angularis cells. Conidiogenous cells discrete, assembled into protruding masses of cells, or integrated in very compact conidiophores. Conidia $4.5-5.5 \times$ 2.5-3.5 $\mu \mathrm{m}(\overline{\mathrm{x}}=4.8 \times 3.2 \mu \mathrm{m}, \mathrm{n}=30)$, ellipsoidal or globose, straight or slightly curved, rounded at both ends, 1-celled, with 1-2 small, guttules, and with thin and smooth walls that are hyaline at secession, becoming light brown and rough-walled.

Known distribution - On Spartium junceum, Italy.

Material examined - ITALY, Arezzo Province, Pieve Santo Stefano, Valsavignone, on dead aerial twigs of Spartium junceum (Fabaceae), 27 May 2012, E. Camporesi IT 384 (MFLU 16-0100, holotype); ITALY, Forlì-Cesena Province, Premilcuore, Fiumicello, on dead aerial branch of Spartium junceum (Fabaceae), 1 April 2012, E. Camporesi IT 208 (MFLU 16-0097).

Notes - Microsphaeropsis is one of the oldest genera in Didymellaceae which was introduced by von Höhnel (1917). The exact familial placement of this genus was uncertain and it has been considered as an asexual morph of Phaeosphaeriaceae (Barr 1987) and Didymosphaeriaceae (Zhang et al. 2012, Thambugala et al. 2017). However, with further morpho-phylo debates, Microsphaeropsis has been referred as a member of Didymellaceae (De Gruyter et al. 2013, Hyde et al. 2013). In a recent study, Chen et al. (2015) reported Microsphaeropsis as a distinct lineage basal to Didymellaceae and the family Microsphaeropsidaceae was introduced. Taxa in Microsphaeropsis produce 'pale greenish brown, finely roughened conidia' (Chen et al. 2015), which differ from most other taxa in Didymellaceae which have mainly hyaline, smooth conidia (phoma-like). Nevertheless, many species of Microsphaeropsis are still unknown from culture or DNA sequence data and Chen et al. (2015), while introducing Microsphaeropsidaceae, recommended that further studies are needed to clarify its precise taxonomic identity and species boundaries.

During our investigation on the diversity of microfungi in Italy, two isolates (MFLU 16-0100, MFLU 16-0097) were recovered from Spartium junceum in Arezzo and Forli-Cesena Provinces. These new isolates share similarities to other Pleosporaceae taxa in their asci and ascospore characteristics, but they share a close phylogenetic affinity to Microsphaeropsis species in our sequence data analyses (Clade A, Fig. 1). However, in this study, Microsphaeropsis species could 
not be segregated from Didymellaceae, in contrast to the results of Chen et al. (2015). Larger datasets of each gene region (ITS, $r p b 2, t u b 2$ ) basically yielded the same major clades as those derived from the concatenated dataset (Fig. 1). Among them, LSU did not provide a better resolution at the generic level and the taxa of Calophoma, Didysimulans, Macroventuria, Microsphaeropsis, Neomicrosphaeropsis, Paraboeremia, Phomatodes and Pseudoascochyta grouped together in an unsupported clade. Although we analysed larger datasets incorporating other family members, we could not find support for segregating Microsphaeropsis from Didymellaceae neither from individual ITS, $r p b 2$ and $t u b 2$ data, nor from concatenated multi-gene analyses. Among the various genes analysed, we noted that $r p b 2$ and $t u b 2$ DNA sequence data yielded rather well-resolved topologies to support intergeneric relationships within Didymellaceae and especially in connection with Microsphaeropsis (data not shown).

Even though the asci and ascospore characters of our new isolates are different from all other Microsphaeropsis species, its asexual morph characteristics are in agreement with the phylogenetic placement, as it has conidia similar to Microsphaeropsis. In concatenated data analyses, our new strains resemble Microsphaeropsis olivacea strains (CBS 233.77, CBS 432.71, CBS 442.83). These strains are however unrelated to any type material and therefore we introduce our new isolates as Microsphaeropsis spartii-juncei sp. nov. Unfortunately, we could not manage to maintain a living culture as subsequent attempts to subculture failed, and hence a living culture is unavailable.

We admit that our phylogeny generated herein does not exactly translate into an appropriate scenario to really demarcate our species but we still recognize it as a different single species occupying a totally different ecological niche. As stated in our paper, there are some degrees of morphological differences in the ascospore characters (despite similarities in conidial characters), which support our new species. However, neither Microsphaeropsis olivacea nor M. proteae have sexual characteristics to compare with $M$. spartii-juncei. Under circumstances where compelling evidence are not available, we follow Jeewon \& Hyde et al. (2016) herein to justify our new species. We note $100 \%$ and $99 \%$ similarity for LSU and ITS in Microsphaeropsis species. There was a 17/334 (5.1\%) difference in the TUB region. There are no RPB2 sequences for Microsphaeropsis olivacea and $M$. proteae. We suspect herein that the genes analysed and the taxon sampling used generating phylogenies could have had an impact and fail to resolve that clade. It is beyond the scope of the study to resolve these. It might also not be a surprise if future discoveries of more species within Microsphaeropsis split the clade and there is a need to segregate one species into several. We have recently witnessed such a phenomenon with Dematiopleospora (Huang et al. 2017). Unless we do some extensive taxonomic reassessment, we would not be tempted to synonymise any extant taxa here.

Neomicrosphaeropsis alhagi-pseudalhagi Wanas., Gafforov \& K.D. Hyde, sp. nov.

Fig. 5 Index Fungorum number: IF554396; Facesoffungi number: FoF 04468

Etymology - Name reflects the host species Alhagi pseudoalhagi, from which the species was isolated.

Holotype - TASM 6134.

Saprobic on Alhagi pseudalhagi (M. Bieb.) Fisch. Sexual morph: Undetermined. Asexual morph: coelomycetous. Conidiomata $150-220 \mu \mathrm{m}$ high $\times 40-70 \mu \mathrm{m}$ diam. $(\overline{\mathrm{x}}=187 \times 52 \mu \mathrm{m}, \mathrm{n}=$ 6), acervuli, hemispherical to spherical, composed of brown to reddish-brown, pseudoparenchymatous cells. Conidiophores reduced to conidiogenous cells. Conidiogenous cells $7-12 \times 8-10 \mu \mathrm{m}(\overline{\mathrm{x}}=10.8 \times 9.1 \mu \mathrm{m}, \mathrm{n}=20)$, holoblastic, phialidic, ampulliform to cylindrical, unbranched, pale brwon, smooth. Conidia 30-45 $\times 18-22 \mu \mathrm{m}(\overline{\mathrm{x}}=37.2 \times 20.7 \mu \mathrm{m}, \mathrm{n}=30)$, variable and irregular, mostly ellipsoidal, terminal, solitary, muriform, 3-5-transversely septate, with 1-3 vertical septa, deeply constricted at the middle septum, slightly constricted at remaining septa, initially pale brown, becoming dark brown at maturity, upper part wider than lower part, rounded at upper end, with flat lower end.

Known distribution - On Alhagi pseudalhagi, Uzbekistan. 

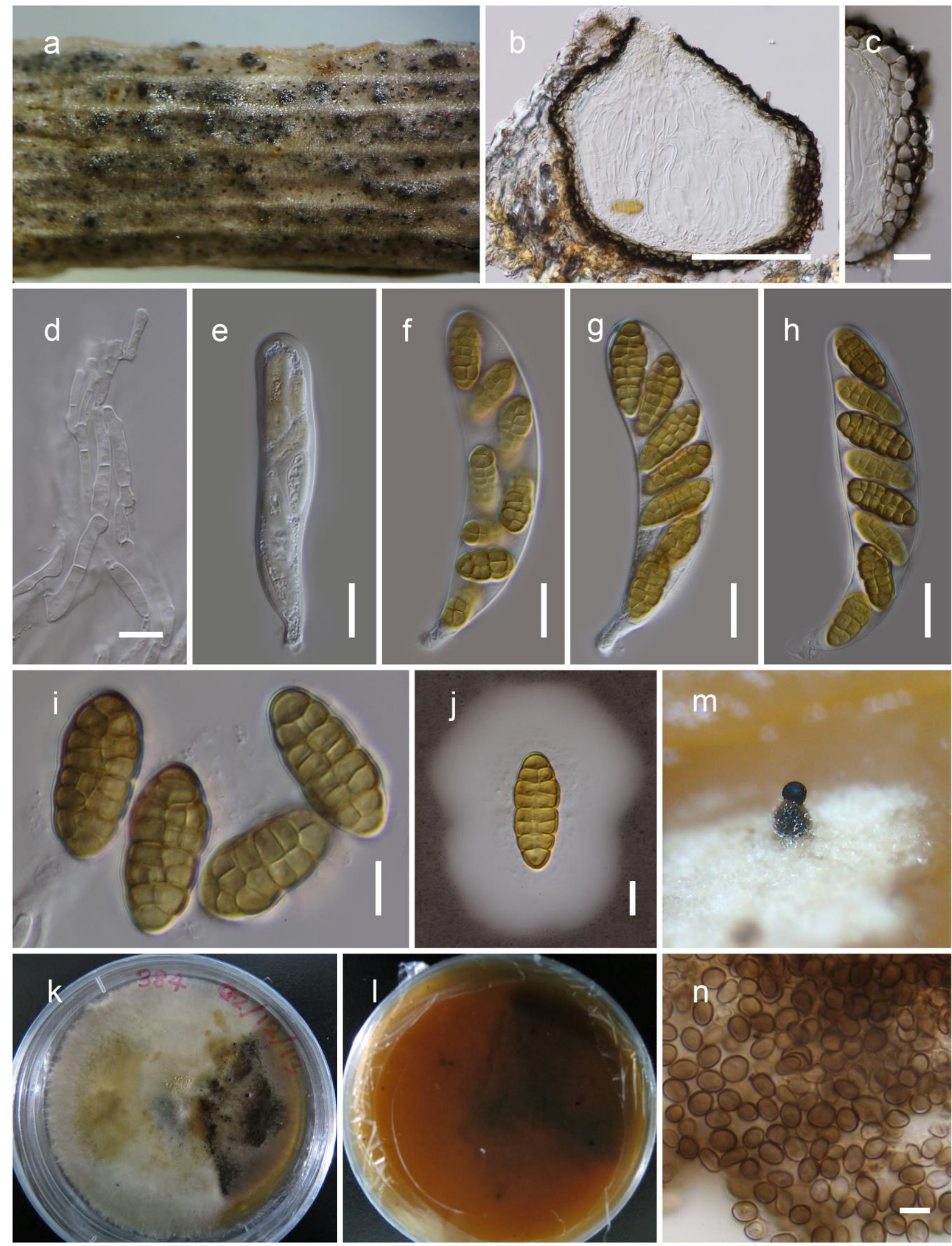

Fig. 4 - Microsphaeropsis spartii-juncei (MFLU 16-0100, holotype). a Appearance of ascomata on host substrate. b Section of ascoma. c Peridium. d Pseudoparaphyses. e-h Asci. i, j Ascospores (Note the ascospore stained with Indian Ink in j). $\mathrm{k}, 1$ Culture on PDA (note 1 reverse). m Conidiama on PDA. $\mathrm{n}$ Conidia. Scale bars: $\mathrm{b}=100 \mu \mathrm{m}, \mathrm{c}, \mathrm{e}-\mathrm{h}=20 \mu \mathrm{m}, \mathrm{d}, \mathrm{i}, \mathrm{j}=10 \mu \mathrm{m}, \mathrm{n}=5$ $\mu \mathrm{m}$. 

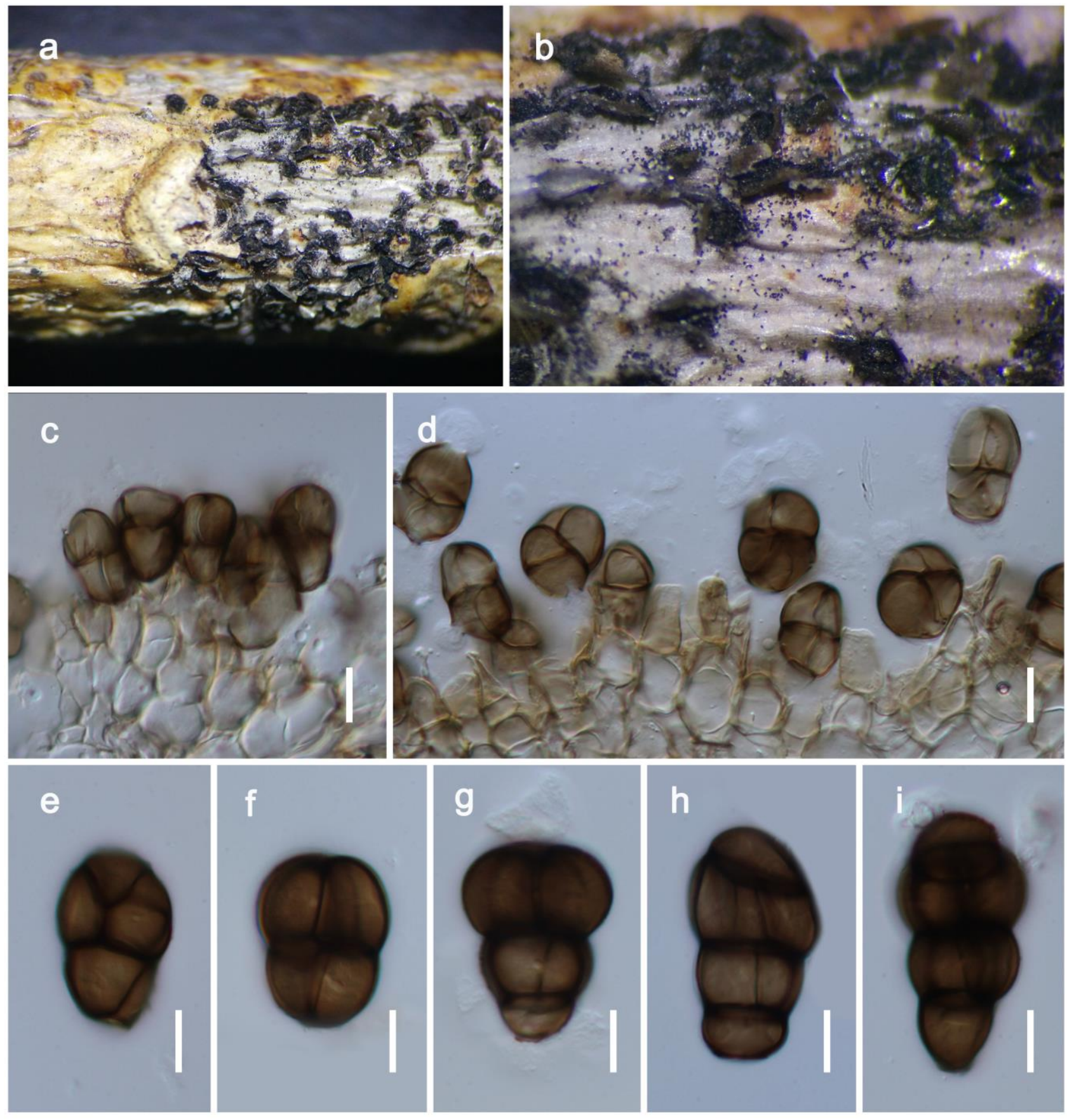

Fig. 5 - Neomicrosphaeropsis alhagi-pseudalhagi (TASM 6134, holotype). a, b Appearance of conidiomata on host substrate. $\mathrm{c}, \mathrm{d}$ Conidia and conidiogenous cells. e-i Conidia. Scale bars: $\mathrm{c}-\mathrm{i}=$ $10 \mu \mathrm{m}$.

Material examined - UZBEKISTAN, Surxondaryo Province, Boysun District, Omonxona Village, South-Western Hissar Mountains, on branches of Alhagi pseudalhagi (Fabaceae), 13 May 2016, Yusufjon Gafforov YG-S24-2 (TASM 6134, holotype; MFLU 17-0190, isotype).

Notes - Neomicrosphaeropsis alhagi-pseudalhagi, collected from Alhagi pseudalhagi in Uzbekistan, is in an independent lineage with good support and phylogenetically distinct from other extant species of Neomicrosphaeropsis (subclade C1, Fig. 1). This new species differs from other taxa in Neomicrosphaeropsis in having acervulus type conidiomata and conidia with 1-3 vertical septa and a deep constriction at the middle septum, whereas other species have pycnidial conidiomata, conidia with 1-2 vertical septa and slight constrictions at their septa. 
Neomicrosphaeropsis cytisicola Wanas., Camporesi, E.B.G. Jones \& K.D. Hyde, sp. nov. $\quad$ Fig. 6 Index Fungorum number: IF554397; Facesoffungi number: FoF 04469

Etymology - Name reflects the host genus Cytisus, from which the species was isolated.

Holotype - MFLU 16-16-0114.

Saprobic on Cytisus sp. Sexual morph: Ascomata 180-250 $\mu \mathrm{m}$ high, $180-220 \mu \mathrm{m}$ diam. $(\overline{\mathrm{x}}=$ $319.6 \times 265.7 \mu \mathrm{m}, \mathrm{n}=5$ ), immersed to semi-erumpent, globose or subglobose, dark brown to black, coriaceous, ostiolate. Ostioles 60-80 long, 100-120 $\mu \mathrm{m}$ wide, apapillate, central, filled with hyaline to brown cells. Peridium 10-15 $\mu \mathrm{m}$ wide at the base, 15-20 $\mu \mathrm{m}$ wide at the sides, comprising reddish to dark brown cells of textura angularis. Hamathecium comprising numerous, $2-2.5 \mu \mathrm{m}$ wide, filamentous, branched, septate, pseudoparaphyses. Asci 140-160 × 30-40 $\mu \mathrm{m}(\overline{\mathrm{x}}=146.6 \times$ $35.6 \mu \mathrm{m}, \mathrm{n}=20$ ), 8 -spored, bitunicate, fissitunicate, clavate, pedicellate, thick-walled at the apex, with minute ocular chamber. Ascospores $32-38 \times 13-18 \mu \mathrm{m}(\overline{\mathrm{x}}=35.8 \times 15.4 \mu \mathrm{m}, \mathrm{n}=30)$, overlapping biseriate, mostly ellipsoidal, muriform, 6-7-transversely septate, with 2-3 vertical septa, slightly constricted at the septa, initially hyaline to pale yellow, becoming brown to dark brown at maturity, narrowly rounded at upper end and rounded at lower end, guttulate, surrounded by a thick mucilaginous sheath (20-30 $\mu \mathrm{m}$ wide). Asexual morph: coelomycetous. Conidiomata superficial or immersed in the agar, pale brown to dark brown, 0.5-1 mm diam, simple, or complex with several merging cavities. Conidiomatal wall composed of textura angularis cells. Conidiophores occasionally present, hyaline, doliiform to ampulliform, arising from inner layers of the pycnidial wall. Conidiogenous cells enteroblastic, phialidic, doliiform or cylindrical to ampulliform, with a periclinal wall thickening at the tip, hyaline, smooth. Conidia 4-7 $\times 2.5-3.5$ $\mu \mathrm{m}(\overline{\mathrm{x}}=5.1 \times 3.1 \mu \mathrm{m}, \mathrm{n}=30)$, ellipsoidal, straight or slightly curved, rounded at both ends, 1 celled, with 1-2 small guttules, and with thin and smooth walls that are hyaline at secession, becoming light brown.

Known distribution - On Cytisus sp., Italy.

Material examined - ITALY, Arezzo Province, Bagno di Cetica, on dead aerial branches of Cytisus sp. (Fabaceae), 1 October 2012, E. Camporesi IT 762 (MFLU 16-0114, holotype); ex-type living culture, MFLUCC 18-0355.

Notes - Neomicrosphaeropsis cytisicola also a novel taxon in this study, which has muriform ascospores, but resembles Laburnicola species in Didymosphaeriaceae more closely than Pleosporaceae taxa in its ascospore characteristics. This novel taxon has closer phylogenetic affinities to Neomicrosphaeropsis cytisi, N. cytisinus and N. minima (subclade C3, Fig. 1). All these mentioned species were isolated from Cytisus and Verbascum species in Italy. Our new species is the first record of sexual morph of taxa in Subclade C3 (Fig. 1) and it differs from the other remaining sexual morph (Neomicrosphaeropsis tamaricicola) in having comparatively larger ascospores $(32-38 \times 13-18 \mu \mathrm{m})$ with more septa $(6-7$ transverse septa, with 2-3 vertical septa), while $N$. tamaricicola has smaller ascospores $(15-20 \times 7-10 \mu \mathrm{m})$ with less septa $(4-6$ transverse septa, with 1 vertical septum). All taxa in Neomicrosphaeropsis produce aseptate brown conidia similar to taxa in Microsphaeropsis including the sexual morph we observed from N. cytisicola sp. nov.

While our two new taxa, Neomicrosphaeropsis alhagi-pseudalhagi and N. cytisicola are wellsupported and resolved, we noted within clade C, where Neomicrosphaeropsis is interspersed, interspecies relationships are obscure. Even our multigene phylogeny fails to properly delineate species in this clade and all species cluster together despite bearing morphological differences. There is a need to redefine species delimitation among these species, possibly using a different approach. In addition, it is also noteworthy to point out taxa in subclade $\mathrm{C} 1$ and $\mathrm{C} 3$ can also be considered as different genera, but until more samples are collected, analysed and typification reevaluated, we refrain from revising the current taxonomic concept.

Neomicrosphaeropsis elaeagni Wanas., Bulgakov, E.B.G. Jones \& K.D. Hyde, sp. nov. Figs 2, 7 Index Fungorum number: IF554398; Facesoffungi number: FoF 04470

Etymology - Name reflects the host genus Elaeagnus, from which the species was isolated. 
Holotype - MFLU 16-2389.

Necrotrophic/saprobic on dying branches of Elaeagnus angustifolia L. Sexual morph: Undetermined. Asexual morph: coelomycetous. Conidiomata pycnidial, 350-400 $\mu \mathrm{m}$ high, $450-550 \mu \mathrm{m} \operatorname{diam}(\overline{\mathrm{x}}=378.7 \times 500.1 \mu \mathrm{m}, \mathrm{n}=10)$, black, superficial to semi-immersed,

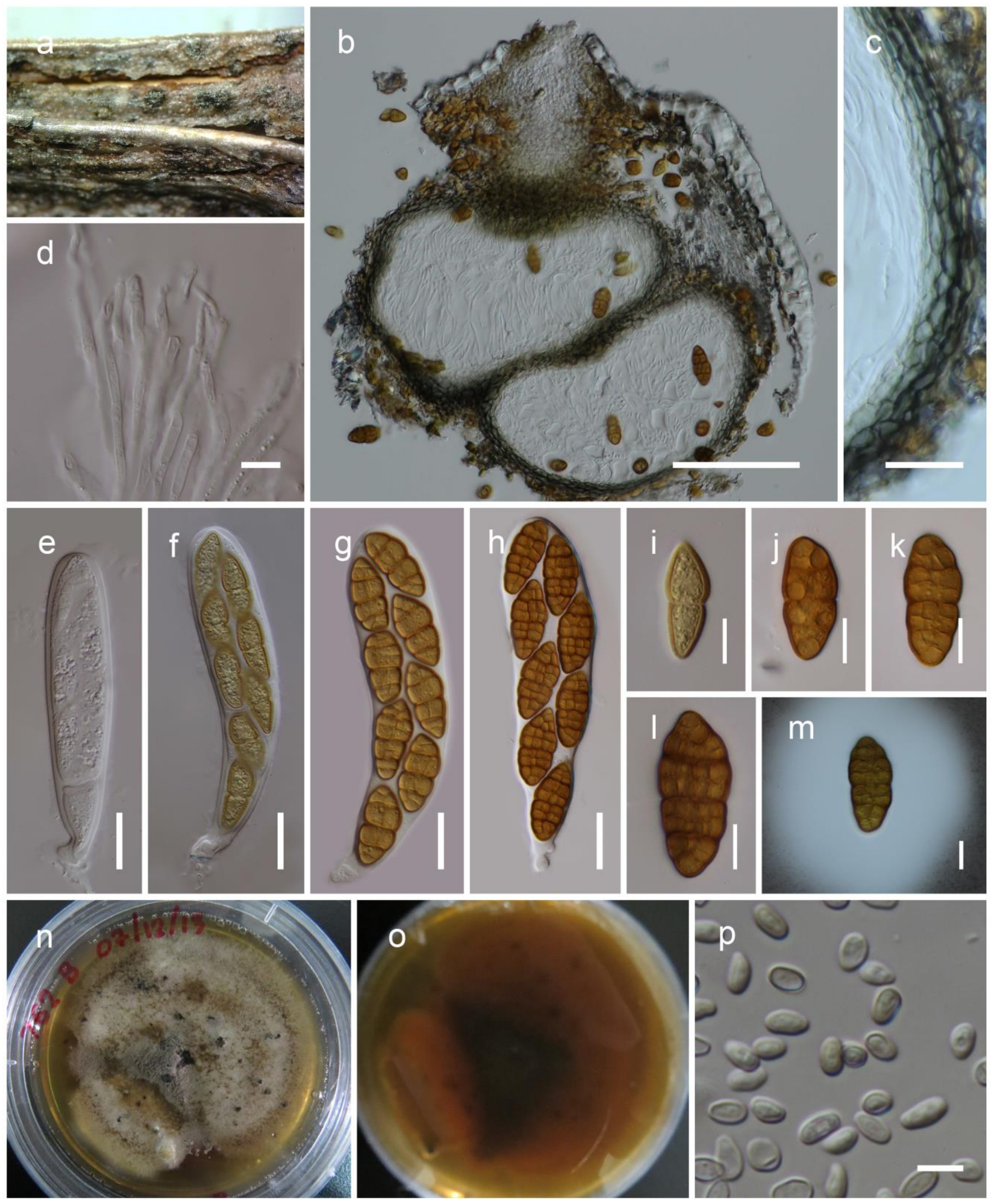

Fig. 6 - Neomicrosphaeropsis cytisicola (MFLU 16-0114, holotype). a Appearance of ascomata on host substrate. b Section of ascomata. c Peridium. d Pseudoparaphyses. e-h Asci. i-m Ascospores (Note the ascospore stained with Indian Ink in $\mathrm{m}$ ). $\mathrm{n}$, o Culture on PDA (note o reverse). $\mathrm{p}$ Conidia. Scale bars: $\mathrm{b}=100 \mu \mathrm{m}, \mathrm{c}, \mathrm{e}-\mathrm{h}=20 \mu \mathrm{m}, \mathrm{d}, \mathrm{i}-\mathrm{m}=10 \mu \mathrm{m}, \mathrm{p}=5 \mu \mathrm{m}$. 

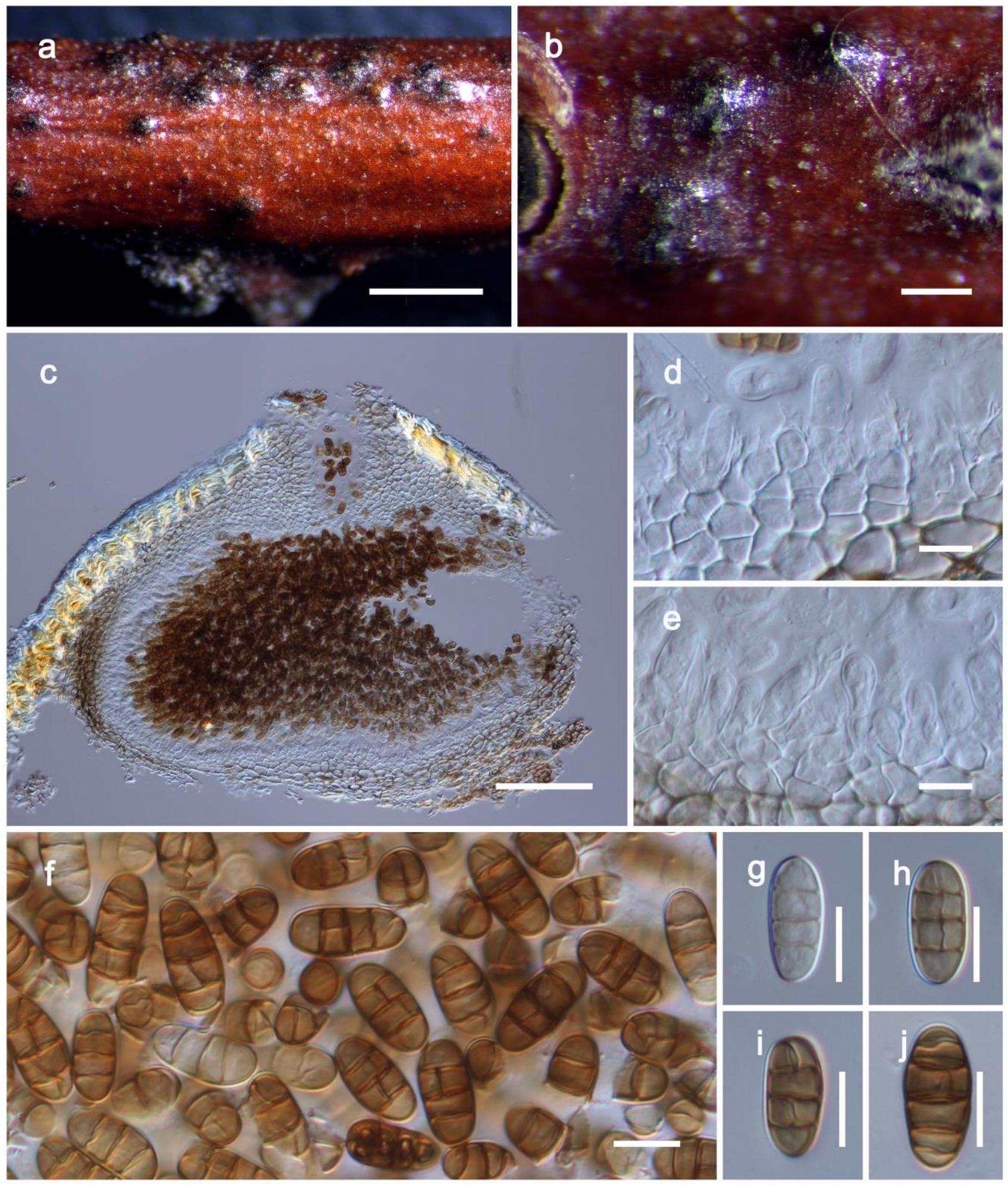

Fig. 7 - Neomicrosphaeropsis elaeagni (MFLU 16-2389, holotype). a Conidiomata on host surface. b Vertical section through conidioma. c Conidiomata wall. $d$, e. Conidiogenous cells producing conidia. $\mathrm{f}-\mathrm{j}$ Conidia. Scale bars: $\mathrm{a}=1 \mathrm{~mm} ; \mathrm{b}=200 \mu \mathrm{m} ; \mathrm{c}=100 \mu \mathrm{m} ; \mathrm{d}-\mathrm{j}=10 \mu \mathrm{m}$.

confluent, gregarious, sometimes scattered beneath the host periderm or on decorticated wood, fully or partly erumpent, globose, ostiolate. Ostiole central, $100-130 \mu \mathrm{m}$ long, $50-80 \mu \mathrm{m}$ diam $(\overline{\mathrm{x}}=$ $117.1 \times 62.7 \mu \mathrm{m}, \mathrm{n}=10$ ), central, long, smooth, sometimes ostiolar canal filled with hyaline or pale brown cells. Pycnidial wall multi-layered, 20-30 $\mu \mathrm{m}$ wide at the base, 30-40 $\mu \mathrm{m}$ wide in sides, thick, comprising two layers, outer layer heavily pigmented, thick-walled, comprising blackish to dark reddish-brown cells of textura angularis, cells towards the inside lighter, inner layer composed 
of hyaline, thin-walled cells of textura angularis. Conidiophores reduced to conidiogenous cells. Conidiogenous cells enteroblastic, annellidic, doliiform, integrated, solitary, hyaline, smoothwalled, and formed from the inner layer of pycnidium wall. Conidia 16-20 $\times 7-9 \mu \mathrm{m}(\overline{\mathrm{x}}=17.5 \times$ $7.7 \mu \mathrm{m} ; \mathrm{n}=50$ ), oblong, straight, rounded at both ends, sometimes narrowly rounded ends, 3-5transversely septate, one longitudinal septum, smooth-walled, initially hyaline, becoming brown to dark brown at maturity.

Known distribution - On Elaeagnus angustifolia, European Russia (Krasnodar region).

Material examined - RUSSIA, Krasnodar region, Novorossiysk, trees near Sudzhuk lagoon (N 44.68114 ${ }^{\circ}$, E $37.79712^{\circ}$ ), on twigs of Elaeagnus angustifolia L. (Elaeagnaceae), 14 June 2016, Timur S. Bulgakov NK-081 (MFLU 16-2389, holotype).

Notes - Neomicrosphaeropsis elaeagni is a novel species which was recovered from Elaeagnus angustifolia in Russia. It was identified as a camarosporium-like taxon by its morphology and further sequence analyses indicate a strong affinity to taxa related to Neomicrosphaeropsis (subclade C1, Fig. 1). Didymellocamarosporium tamaricis also clusters in this clade as another camarosporium-like species. Wijayawardene et al. (2016) proposed Didymellocamarosporium as a monotypic genus based on rDNA sequence data available from GenBank for the type, D. tamaricis. Both Neomicrosphaeropsis elaeagni and Didymellocamarosporium tamaricis are morphologically similar in their conidiomata, conidiogenous cells and conidial characteristics. However, taxa in this subclade $\mathrm{C} 1$ are heterogenous and we could not demarcate Didymellocamarosporium and Neomicrosphaeropsis into two separate genera from our multi-gene phylogenetic analyses. It is therefore necessary to collect more fungi similar to Didymellocamarosporium and Neomicrosphaeropsis in different geographic regions, isolate them into culture, describe their morphology, analyse their DNA sequences and investigate their phylogenetic relationships to better identify and classify them.

\section{Acknowledgements}

Dhanushka Wanasinghe would like to thank the Molecular Biology Experimental Center at Kunming Institute of Botany for facilities for molecular work. We thank Pranami Abeywickrama for her valuable assistance. Shaun Pennycook is thanked for nomenclatural advices. Rajesh Jeewon thanks the University of Mauritius and Mae Fah Luang University for research support. Yusufjon Gafforov acknowledges the Committee for Coordination Science and Technology Development under the Cabinet of Ministers of Uzbekistan for research support (\#P3-2014-0830174425).

\section{References}

Ariyawansa HA, Hyde KD, Jayasiri SC, Buyck B et al. 2015 - Fungal diversity notes 111-252 taxonomic and phylogenetic contributions to fungal taxa. Fungal Diversity 75, 27-274.

Aveskamp MM, De Gruyter J, Crous PW. 2008 - Biology and recent developments in the systematics of Phoma, a complex genus of major quarantine significance. Fungal Diversity $31,1-18$.

Aveskamp MM, De Gruyter J, Woudenberg JHC, Verkley GJM et al. 2010 - Highlights of the Didymellaceae: a polyphasic approach to characterise Phoma and related pleosporalean genera. Studies in Mycology 65, 1-60.

Barr ME. 1987 - Prodromus to Class Loculoascomycetes. Published by the Author, Amherst, Massachusetts; University of Massachusetts, U.S.A.

Chen Q, Hou LW, Crous PW, Cai L. 2017 - Didymellaceae revisited. Studies in Mycology 87, 105-159.

Chen Q, Jiang JR, Zhang GZ, Cai L et al. 2015 - Resolving the Phoma enigma. Studies in Mycology 82, 137-217.

Crous PW, Summerell BA, Swart L, Denman S et al. 2011 - Fungal pathogens of Proteaceae. Persoonia 27, 20-45. 
Crous PW, Wingfield MJ, Burgess TI, Hardy GESt J. 2016 - Fungal Planet description sheets: 469-557. Persoonia 37, 218-403.

Crous PW, Wingfield MJ, Schumacher RK, Summerell BA et al. 2014 - Fungal planet description sheets: 281-319. Persoonia 33, 212-289

De Gruyter J, Aveskamp MM, Woudenberg JHC, et al. 2009 - Molecular phylogeny of Phoma and allied anamorph genera: towards a reclassification of the Phoma complex. Mycological Research 113, 508-519.

De Gruyter J, Woudenberg JHC, Aveskamp AA, Verkley GJM, et al. 2013 - Redisposition of Phoma-like anamorphs in Pleosporales. Studies in Mycology 75, 1-36.

Hall TA. 1999 - BioEdit: a user-friendly biological sequence alignment editor and analysis program for Windows 95/98/NT. In: Nucleic Acids Symposium Series 41, 95-98.

Hyde KD, Jones EBG, Liu JK, Ariyawansa H et al. 2013 - Families of Dothideomycetes. Fungal Diversity 63, 1-313.

Huang S, Jeewon R, Wanasinghe DN, Manawasinghe IS et al. 2017 - Phylogenetic taxonomy of Dematiopleospora fusiformis sp. nov. (Phaeosphaeriaceae) from Russia. Phytotaxa, 316, 239249.

Index Fungorum. 2018 - http://www.indexfungorum.org/Names/Names.asp.

Jayasiri SC, Hyde KD, Ariyawansa HA, Bhat J et al. 2015 - The Faces of Fungi database: fungal names linked with morphology, phylogeny and human impacts. Fungal Diversity 74, 3-18.

Jayasiri SC, Hyde KD, Jones EBG, Jeewon R et al. 2017 - Taxonomy and multigene phylogenetic evaluation of novel species in Boeremia and Epicoccum with new records of Ascochyta and Didymella (Didymellaceae). Mycosphere 8, 1080-1101.

Jeewon R, Hyde KD. 2016 - Establishing species boundaries and new taxa among fungi: recommendations to resolve taxonomic ambiguities. Mycosphere 7, 1669-1677.

Jeewon R, Ittoo J, Mahadeb D, Jaufeerally-Fakim Y et al. 2013 - DNA based identification and phylogenetic characterisation of endophytic and saprobic fungi from Antidesma madagascariense, a medicinal plant in mauritius. Journal of Mycology 2013, 1-10.

Jeewon R, Liew E, Hyde K. 2004 - Phylogenetic evaluation of species nomenclature of Pestalotiopsis in relation to host association. Fungal Diversity 17, 39-55.

Jeewon R, Liew ECY, Hyde KD. 2003a - Molecular systematics of the Amphisphaeriaceae based on cladistic analyses of partial LSU rDNA gene sequences. Mycological Research 107, 1392 1402.

Jeewon R, Liew EC, Simpson JA, Hodgkiss IJ et al. 2003b - Phylogenetic significance of morphological characters in the taxonomy of Pestalotiopsis species. Molecular Phylogenetics and Evolution 27, 372-383.

Katoh k, Rozewicki J, Yamada KD. 2017 - MAFFT online service: multiple sequence alignment, interactive sequence choice and visualization, Briefings in Bioinformatics, bbx108, https://doi.org/10.1093/bib/bbx108

Kishino H, Hasegawa M. 1989 - Evaluation of the maximum likelihood estimate of the evolutionary tree topologies from DNA sequence data, and the branching order in hominoidea. Journal of Molecular Evolution 29, 170-179.

Kuraku S, Zmasek CM, Nishimura O, Katoh K. 2013 - aLeaves facilitates on-demand exploration of metazoan gene family trees on MAFFT sequence alignment server with enhanced interactivity. Nucleic Acids Res. 41(Web Server issue), W22-W28.

Liu JK, Hyde KD, Jeewon R, Phillips AJ et al. 2017 - Ranking higher taxa using divergence times: a case study in Dothideomycetes. Fungal Diversity 84, 75-99.

Liu YJ, Whelen S, Hall BD. 1999 - Phylogenetic relationships among ascomycetes evidence from an RNA polymerase II subunit. Molecular Biology and Evolution 16, 1799-1808.

Miller MA, Pfeiffer W, Schwartz T. 2010 - Creating the CIPRES Science Gateway for inference of large phylogenetic trees. In: Proceedings of the gateway computing environments workshop (GCE). Institute of Electrical and Electronics Engineers, New Orleans, LA, 14 Nov, pp 1-8.

MycoBank. 2017 - http://www.mycobank.org/quicksearch.aspx. 
Nylander JAA. 2004 - MrModeltest 2.0. Program distributed by the author. Evolutionary Biology Centre, Uppsala University.

Rambaut A, Drummond AJ. 2007 - Tracer v1, 4. Available from: http://beast.bio.ed.ac.uk/Tracer.

Rambaut A. 2012 - FigTree version 1.4.0. Available at http://tree.bio.ed.ac.uk/software/figtree/

Rehner SA, Samuels GJ. 1994 - Taxonomy and phylogeny of Gliocladium analysed from nuclear large subunit ribosomal DNA sequences. Mycological Research 98, 625-634.

Ronquist F, Teslenko M, van der Mark P, Ayres DL et al. 2012 - MrBayes 3.2: Efficient Bayesian phylogenetic inference and model choice across a large model space. Systematic Biology 61, 539-542.

Stamatakis A 2014 - RAxML version 8: a tool for phylogenetic analysis and post-analysis of large phylogenies. Bioinformatics 30, 1312-1313.

Stamatakis A, Hoover P, Rougemont J. 2008 - A rapid bootstrap algorithm for the RAxML web servers. Systematic Biology 57, 758-771.

Sung GH, Sung JM, Hywel-Jones NL, Spatafora JW. 2007 - A multi-gene phylogeny of Clavicipitaceae (Ascomycota, Fungi): identification of localized incongruence using a combinational bootstrap approach. Molecular Phylogenetics and Evolution 44, 1204-1223.

Thambugala KM, Daranagama DA, Phillips AJL, Balgakov TS et al. 2017- Microfungi on Tamarix. Fungal Diversity 82, 239-306.

Tibpromma S, Hyde KD, Jeewon R, Maharachchikumbura SSN et al. 2017 - Fungal diversity notes 491-602: taxonomic and phylogenetic contributions to fungal taxa. Fungal Diversity 83, 1261.

Valenzuela-Lopez N, Cano-Lira JF, Guarro J, Sutton DA et al. 2018 - Coelomycetous Dothideomycetes with emphasis on the families Cucurbitariaceae and Didymellaceae. Studies in Mycology 90, 1-69.

Verkley GJM, Dukik K, Renfurm R, Göker M et al. 2014 - Novel genera and species of coniothyrium-like fungi in Montagnulaceae (Ascomycota). Persoonia 32, 25-51.

Vilgalys R, Hester M. 1990 - Rapid genetic identification and mapping of enzymatically amplified ribosomal DNA from several Cryptococcus species. Journal of Bacteriology 172, 4238-4246.

Von Höhnel F. 1917 - Fungi imperfecti. Beiträge zur Kenntnis derselben Hedwigia 59, 236-284.

Wanasinghe DN, Hyde KD, Jeewon R, Crous et al. 2017a - Phylogenetic revision of Camarosporium (Pleosporineae, Dothideomycetes) and allied genera. Studies in Mycology 87, 207-256.

Wanasinghe DN, Jones EBG, Camporesi E, Boonmee S et al. 2014a - An exciting novel member of Lentitheciaceae in Italy from Clematis vitalba. Cryptogamie Mycologie 35, 323-337.

Wanasinghe DN, Jones EBG, Camporesi E, Boonmee S et al. 2014b - Dematiopleospora mariae gen sp nov from Ononis spinosa in Italy. Cryptogamie Mycologie 35, 105-117.

Wanasinghe DN, Jones EBG, Camporesi E, Dissanayake AJ et al. 2016 - Taxonomy and phylogeny of Laburnicola gen. nov. and Paramassariosphaeria gen. nov. (Didymosphaeriaceae, Massarineae, Pleosporales). Fungal Biology 120, 1354-1373.

Wanasinghe DN, Jones EG, Camporesi E, Mortimer PE et al. 2015 - The genus Murispora. Cryptogamie Mycologie 36, 419-448.

Wanasinghe DN, Phookamsak R, Jeewon R, Li WJ et al. 2017b - A family level rDNA based phylogeny of Cucurbitariaceae and Fenestellaceae with descriptions of new Fenestella species and Neocucurbitaria gen. nov. Mycosphere 8, 397-414.

Wanasinghe DN, Phukhamsakda C, Hyde KD, Jeewon R et al. 2018 - Fungal diversity notes 709839: taxonomic and phylogenetic contributions to fungal taxa with an emphasis on fungi on Rosaceae. Fungal Diversity 89, 1-238.

White TJ, Bruns T, Lee J, Taylor SB. 1990 - Amplification and direct sequencing of fungal ribosomal RNA genes for phylogenetics. In: Innis MA, Gelfand DH, Sninsky JJ, White TJ (eds), PCR protocols: a guide to methods and applications: 315-322. Academic Press, San Diego, California, USA. 
Wijayawardene NN, Hyde KD, Lumbsch HT, Liu JK et al. 2018 - Outline of Ascomycota: 2017 Fungal Diversity 88, 167-263.

Wijayawardene NN, Hyde KD, Wanasinghe DN, Papizadeh M et al. 2016 - Taxonomy and phylogeny of dematiaceous coelomycetes. Fungal Diversity 77, 1-316.

Woudenberg JHC, Aveskamp MM, De Gruyter J, Spiers AG et al. 2009 - Multiple Didymella teleomorphs are linked to the Phoma clematidina morphotype. Persoonia 22, 56-62.

Zhang Y, Crous PW, Schoch CL, Hyde KD. 2012 - Pleosporales. Fungal Diversity 53, 1-221. 